Subscriber access provided by Caltech Library

\title{
Article
}

\section{Discrete Dimers of Redox-Active and Fluorescent Perylene Diimide-Based Rigid Isosceles Triangles in the Solid State}

Siva Krishna Mohan Nalluri, Jiawang Zhou, Tao Cheng, ZHICHANG LIU, Minh T. Nguyen, Tianyang Chen, Hasmukh A. Patel, Matthew D. Krzyaniak, William A. Goddard, Michael R. Wasielewski, and J. Fraser Stoddart

J. Am. Chem. Soc., Just Accepted Manuscript • DOI: 10.1021/jacs.8b11201 • Publication Date (Web): 11 Dec 2018

Downloaded from http://pubs.acs.org on December 12, 2018

\section{Just Accepted}

"Just Accepted" manuscripts have been peer-reviewed and accepted for publication. They are posted online prior to technical editing, formatting for publication and author proofing. The American Chemical Society provides "Just Accepted" as a service to the research community to expedite the dissemination of scientific material as soon as possible after acceptance. "Just Accepted" manuscripts appear in full in PDF format accompanied by an HTML abstract. "Just Accepted" manuscripts have been fully peer reviewed, but should not be considered the official version of record. They are citable by the Digital Object Identifier (DOI®). "Just Accepted" is an optional service offered to authors. Therefore, the "Just Accepted" Web site may not include all articles that will be published in the journal. After a manuscript is technically edited and formatted, it will be removed from the "Just Accepted" Web site and published as an ASAP article. Note that technical editing may introduce minor changes to the manuscript text and/or graphics which could affect content, and all legal disclaimers and ethical guidelines that apply to the journal pertain. ACS cannot be held responsible for errors or consequences arising from the use of information contained in these "Just Accepted" manuscripts. 


\section{Discrete Dimers of Redox-Active and Fluorescent Perylene Diimide-Based Rigid Isosceles Triangles in the Solid State}

Siva Krishna Mohan Nalluri, ${ }^{\dagger}$ Jiawang Zhou, ${ }^{\dagger, \star}$ Tao Cheng, $\|$ Zhichang Liu, ${ }^{\dagger}$ Minh T. Nguyen, ${ }^{\dagger}$ Tianyang Chen, ${ }^{\dagger}$ Hasmukh A. Patel, ${ }^{\dagger}$ Matthew D. Krzyaniak, ${ }^{\dagger}$ William A. Goddard III, $\|$ Michael R. Wasielewski ${ }^{*}, \dagger$, and J. Fraser Stoddart ${ }^{*}, \dot{\dagger},, \#$

${ }^{\dagger}$ Department of Chemistry, Northwestern University, 2145 Sheridan Road, Evanston, Illinois 60208, USA

*Institute for Sustainability and Energy at Northwestern, Northwestern University, 2145 Sheridan Road, Evanston, Illinois 60208, USA

॥ Materials and Process Simulation Center, California Institute of Technology, Pasadena, California 91125 , USA

$\S$ Institute for Molecular Design and Synthesis, Tianjin University, 92 Weijin Road, Nankai District, Tianjin 300072, China.

\# School of Chemistry, University of New South Wales, Sydney, NSW 2052, Australia.

*E-mail: m-wasielewski@northwestern.edu; stoddart@northwestern.edu.

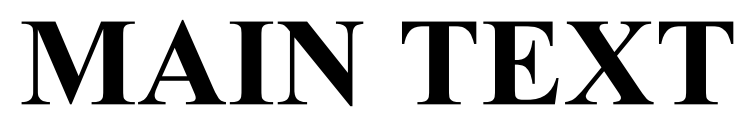

\begin{tabular}{|c|}
\hline *Correspondence Address \\
\hline Professor J. Fraser Stoddart \\
Department of Chemistry \\
Northwestern University \\
2145 Sheridan Road \\
Evanston, IL 60208 (USA) \\
Email: stoddart@northwestern.edu \\
\hline
\end{tabular}




\section{- ABSTRACT}

The development of rigid covalent chiroptical organic materials, with multiple, readily available redox states, which exhibit high photoluminescence is of particular importance in relation to both organic electronics and photonics. The chemically stable, thermally robust and redox-active perylene diimide (PDI) fluorophores have received ever-increasing attention owing to their excellent fluorescence quantum yields in solution. Planar PDI derivatives, however, generally suffer from aggregation-caused emission quenching in the solid state. Herein, we report on the design and synthesis of two chiral isosceles triangles wherein one PDI fluorophore and two pyromellitic diimide (PMDI) or naphthalene diimide (NDI) units are arranged in a rigid cyclic triangular geometry. The optical, electronic and magnetic properties of the rigid isosceles triangles are fully characterized by a combination of optical spectroscopies, X-ray diffraction, cyclic voltammetry, and computational modeling techniques. Single-crystal X-ray diffraction analysis shows that both isosceles triangles form discrete, nearly cofacial PDI-PDI $\pi$-dimers in the solid state. While the triangles exhibit fluorescence quantum yields of almost unity in solution, the dimers in the solid state exhibit very weak — yet at least an order of magnitude higher — excimer fluorescence yield in comparison with the almost completely quenched fluorescence of a reference PDI. The triangle containing both NDI and PDI subunits shows superior intramolecular energy transfer from the lowest excited singlet state of the NDI to that of the PDI subunit. Cyclic voltammetry suggests that both isosceles triangles exhibit multiple, easily accessible and reversible redox states. Applications beckon in arenas related to molecular optoelectronic devices. 


\section{- INTRODUCTION}

The molecular design and development of redox-active organic materials displaying efficient photoluminescence (PL) is a fundamental prerequisite for the fabrication of optoelectronic and photonic devices. ${ }^{1-8}$ Organic fluorophores, exhibiting intense fluorescence in dilute solution, often suffer from emission quenching ${ }^{9-10}$ in the solid state because of strong intermolecular interactions involving many molecules. Although it is often difficult to predict the fluorescence quantum yield of a particular fluorophore, several strategies ${ }^{11-21}$ have been proposed in the literature to enhance their fluorescence efficiency. These strategies include (i) the introduction of bulky substituents into the parent fluorophore to prevent the detrimental intermolecular interactions between the neighboring fluorophores, ${ }^{11-14}$ (ii) the restriction of intramolecular rotations of the fluorophore side groups to minimize radiationless deactivation, (iii) the enforcement of a conformational change from a twisted conformation in solution to a planar one in the solid state, ${ }^{15}$ (iv) the formation of $J$ type fluorophore aggregates, ${ }^{16}$ and (v) the enhancement of intramolecular charge transfer (ICT) character within donor- $\pi$-acceptor systems. ${ }^{17-18}$ Also, the development of chiral emissive organic molecules exhibiting circularly polarized luminescence (CPL) has attracted increasing attention in the recent past. ${ }^{22-23}$ Despite these significant advances, the development of novel molecular designs for rigid covalent chiral organic materials with multiple, readily available reversible redox states, exhibiting photoluminescence with high quantum yields both in solution and solid state, remains a formidable challenge. ${ }^{24}$

In the past two decades, perylene diimide (PDI) derivatives have emerged as one of the most extensively investigated model organic fluorophores because of their near unity fluorescence quantum yields in solution in addition to their excellent chemical, thermal and photochemical stabilities. ${ }^{25-27}$ The unique redox-active characteristics associated with the high electron mobilities 
of PDIs renders them attractive candidates ${ }^{28-29}$ for applications in a wide variety of domains, such as organic light-emitting diodes ${ }^{30}$ (OLEDs), organic light-emitting field-effect transistors ${ }^{31-32}$ (OFETs) and organic photovoltaics ${ }^{33-35}$ (OPVs). Selective substitution at the core positions of the PDI fluorophores has been well-established ${ }^{36-38}$ as a means of improving their optical properties. The fluorescence of PDIs is quenched in the solid state on account of their planarity which favors mostly $H$-type aggregation by dint of consecutive $\pi$ - $\pi$ interactions and/or dipole-dipole interactions between the neighboring fluorophores. ${ }^{39-40}$ Nevertheless, Würthner and co-workers ${ }^{41-42}$ have shown that the introduction of bulky substituents onto the PDI fluorophore is an effective strategy to favor the formation of discrete PDI-PDI $\pi$-dimers and undistorted planar PDI fluorophores, ${ }^{43-44}$ which inhibit long-range PDI aggregation in the solid state, giving rise to variable structural and optical properties, depending on the nature of the substituents. It is noteworthy that the conformational flexibility offered by the core- or bay-substituents on the PDI fluorophores in most cases, however, leads to inevitable intramolecular rotations or twists, which presumably reduce their fluorescence quantum yields as a result of enhanced nonradiative decay. ${ }^{45-46}$ Therefore, we envision that the incorporation of a core- or bay-unsubstituted PDI fluorophore - with solubilizing bulky and chiral substituents at the imide positions - into a rigid cyclic geometry that blocks one side of the PDI plane would not only prevent long-range intermolecular interactions but would restrict also intramolecular rotations.

In the past few years, we have focused our attention on the design, synthesis, characterization and applications of a series of enantiopure chiral rigid diimide-based oligomeric macrocycles, ${ }^{47}$ including dimers, ${ }^{48-49}$ trimers, ${ }^{50-56}$ and tetramers, ${ }^{57}$ of which the cyclic trimers are observed to be particularly attractive. ${ }^{47}$ Initially, we reported ${ }^{50}$ a rigid equilateral triangle (-)-3NDI- $\Delta$, comprising three equivalent naphthalene diimide (NDI) units which gave rise to six individually accessible 
one-electron redox states. Extensive characterization of the symmetric (-)-3NDI- $\Delta$ by singlecrystal X-ray diffraction revealed ${ }^{51,54}$ its distinct packing arrangements in the solid state, depending on the nature of its encapsulated guest ${ }^{50}$ and solvent ${ }^{51}$ molecules, as well as its chirality ${ }^{54}$ and electronic state. ${ }^{56,58-59}$ In summary, the assembly of (-)-3NDI- $\Delta$ resulted in the formation of (i) one-dimensional helical superstructures driven by anion- $\pi$ induced face-to-face $\pi$ $\pi$ stacking of two of the NDI units of (-)-3NDI- $\Delta$ in the presence of an encapsulated linear $\mathrm{I}_{3}^{-}$ anion in $\mathrm{CHCl}_{3}$, as well as (ii) finite and infinite supramolecular nanotubes in the presence of encapsulated dihaloethane and -ethene (DXE) driven by the columnar stacking of (-)-3NDI- $\Delta$ with cooperative weak $[\mathrm{C}-\mathrm{H} \cdots \mathrm{O}]$ interactions along the direction of $[\mathrm{X} \cdots \mathrm{X}]$-bonded solvent chains. On the other hand, the monoradical anion $\mathbf{3} \mathbf{N D I}^{--}$assembled into a $K_{4}$ structure $^{58}$ driven by the intermolecular face-to-face $\pi-\pi$ stacking interactions between the NDI radical anions in the solid state, while the trisradical trianion $\mathbf{3} \mathbf{N D I}^{3(\cdot-)}$ strongly associated with three cobaltocenium $\left(\mathrm{CoCp}_{2}{ }^{+}\right)$cations assembled into infinite one-dimensional channels ${ }^{56}$ by dint of electrostatic and hydrogen bonding interactions.

Furthermore, we recently reported two isosceles triangles ${ }^{55}[(-)-2$ PMDI-1NDI- $\Delta$ and (-)2NDI-1PMDI- $\Delta$ ], which are obtained by replacing one of the redox-active units with another in the case of the equilateral triangles $\left[(-)-3\right.$ PMDI- $\Delta^{60}$ and $(-)-3$ NDI- $\left.\Delta\right]$ without disrupting the triangular geometry. Unlike the equilateral triangles, these isosceles triangles have lower symmetries which lack the ability to form extended 1D tubular (super)structures but give rise to 2D layer-like (super)structures in the solid state. Based on the knowledge gained from the intramolecular cyclical through-space electron sharing properties and the distinct solid-state packing arrangements associated with all of the PMDI- and NDI-based trimers, we now intend to include the PDI derivatives in these cyclic systems in order to achieve efficient optoelectronic and 
photonic properties in the realm of small-molecule organic materials. Considering the synthetic challenges associated with the poor solubility of core- or bay-unsubstituted PDI fluorophores, we recently reported ${ }^{52}$ the one-pot synthesis of a molecular triangle composed of three bulky $1,6,7,12-$ tetra(phenoxy)-substituted PDIs. Unlike the previously reported rigid molecular triangles to date, this PDI-based equilateral triangle containing as many as 12 flexible phenoxy substituents could neither be crystallized nor encapsulate suitable guest molecules. Surprisingly, we found that the fluorescence emission of this PDI triangle is quenched, even in dilute $\mathrm{CH}_{2} \mathrm{Cl}_{2}$ with a fluorescence quantum yield $\left(\Phi_{\mathrm{f}}\right)$ of about $0.2 \%$, as a result of highly efficient nonradiative decay by means of an ultrafast photoinduced intramolecular symmetry-breaking charge separation process. In contrast with the six distinct one-electron redox waves observed for the PMDI- or NDI-based equilateral triangles, cyclic voltammetry reveals only two distinct reversible reduction waves, involving a total of six electrons for this PDI triangle. Therefore, we anticipated that the design and synthesis of rigid core- or bay-unsubstituted PDI-based isosceles, rather than equilateral, triangles would be of particular value in an attempt to improve the structural, optical, electronic and magnetic properties of the diimide-based triangles. These unique rigid chiral cyclic systems may serve as model platforms for the investigation of (i) the through-space electron communication as well as of (ii) the solid-state packing arrangements with respect to the competitive intermolecular $\pi-\pi$ stacking interactions between the non-identical redox-active units, with different dimensions, present in confined environments.

Herein, we report the design, synthesis and the full characterization of two chiral rigid $\mathrm{N}$ substituted PDI-based isosceles triangles — namely, (-)-2PMDI-1PDI- $\Delta$ and (-)-2NDI-1PDI- $\Delta$ — in both solution and solid states, and compare the results with those of the related monomeric reference compounds, Ref-PMDI, ${ }^{61}$ Ref-NDI ${ }^{62}$ and Ref-PDI. ${ }^{63}$ The properties of the isosceles 
triangles are characterized by a combination of spectroscopies [steady-state and transient absorption, fluorescence, circular dichroism (CD), electron paramagnetic resonance (EPR), electron-nuclear double resonance (ENDOR)], variable temperature powder X-ray diffraction (VT-PXRD), single-crystal X-ray diffraction (XRD), thin film XRD, cyclic voltammetry (CV) and computational modeling techniques. XRD Analysis shows that the rigid triangular geometry of the macrocycles suppresses the conventional global $\pi-\pi$ stacking into discrete nearly cofacial PDI-PDI $\pi$-dimers. The optical properties of the compounds have been investigated by steady-state and transient absorption, and fluorescence spectroscopies both in solution and solid state. Although the fluorescence quantum yields of the isosceles triangles are almost unity in solution, they exhibit very weak excimer emission in the solid state when compared to the almost completely quenched photoluminescence of the monomeric Ref-PDI. The electronic properties investigated by CV suggest that the isosceles triangles exhibit multiple reversible redox states implicating a total of up to six electrons. The magnetic properties studied by EPR and ENDOR spectroscopies, supported by density functional theory calculations, indicate that the behavior of the unpaired electron on the singly reduced PDI subunit is indeed dependent on the nature of the adjacent PMDI/NDI subunits present within the isosceles triangles.

\section{- RESULTS AND DISCUSSION}

Syntheses of PDI-based isosceles triangles. In contrast with the synthesis of the PMDI/NDIbased isosceles triangles reported ${ }^{55}$ recently by us, here we outline the synthesis of the rigid cyclic PMDI/NDI/PDI trimers involving non-identical redox-active aromatic subunits with different dimensions. The two rigid PDI-based isosceles triangles were prepared (Figure 1) by stepwise condensations between commercially available $(R R)$-trans-1,2-cyclohexanediamine and three 
different dianhydride derivatives - namely, pyromellitic dianhydride (PMDA), naphthalenetetracarboxylic dianhydride (NDA) and perylenetetracarboxylic dianhydride (PDA). Starting with the condensation between $(R R)$-trans-1,2-cyclohexanediamine and an excess of either PMDA in $\mathrm{AcOH}$ at $120{ }^{\circ} \mathrm{C}$ or NDA in DMF at $140{ }^{\circ} \mathrm{C}$ gave the corresponding monoimide-monoanhydride dimers-namely, (-)-2PMIA ${ }^{55}$ and (-)-2NIA ${ }^{57}$-as previously reported by us. The subsequent condensation of the dimers with an excess of mono- $N$-Boc- $(R R)-$ trans-1,2-cyclohexanediamine in DMF at $130{ }^{\circ} \mathrm{C}$ afforded their carbamate derivatives, (-)2PMDI-2NHBoc and (-)-2NDI-2NHBoc, respectively, in good yields. Removal of the Boc groups using trifluoroacetic acid (TFA) gave the corresponding diamines, (-)-2PMDI-2 $\mathbf{N H}_{\mathbf{2}}$ and (-)-2NDI-2NH $\mathrm{N}_{2}$. The cyclocondensation of these diamines with 1 mol equiv of PDA in the presence of $\mathrm{Zn}(\mathrm{OAc})_{2}$ in molten imidazole at $140{ }^{\circ} \mathrm{C}$ afforded (Figure 1) the desired highly rigid isosceles triangles (-)-2PMDI-1PDI- $\Delta$ and (-)-2NDI-1PDI- $\Delta$ in 31 and 22\% yields, respectively. See the Supporting Information for more details.

Detection of peaks at $m / z=1063.2917$ and 1163.3213 in the gas phase for $[M+\mathrm{H}]^{+}$ions by electrospray ionization high-resolution mass spectrometry (ESI-HRMS) confirmed the existence of both (-)-2PMDI-1PDI- $\Delta$ and (-)-2NDI-1PDI- $\Delta$, respectively. Both the ${ }^{1} \mathrm{H}$ and ${ }^{13} \mathrm{C}$ NMR spectra (Figure 2 and Figures S1-S5) of the isosceles triangles (-)-2PMDI-1PDI- $\Delta$ and (-)2NDI-1PDI- $\Delta$ were in agreement with them having rigid cyclic structures with lower symmetry ( $C_{2}$ point group) when compared to the higher symmetry ( $D_{3}$ point group) of the equilateral triangles, (-)-3NDI- $\Delta$ and (-)-3PMDI- $\Delta$. The assignments of all of the resonances corresponding to aromatic, methine and methylene protons of the triangles were confirmed by two-dimensional ${ }^{1} \mathrm{H}-{ }^{1} \mathrm{H}$ and ${ }^{1} \mathrm{H}-{ }^{13} \mathrm{C}$ correlation spectroscopies. In particular, the ${ }^{1} \mathrm{H}$ NMR spectrum of (-)-2PMDI1PDI- $\Delta$ shows (Figure 2 and Figure S1) three sets of signals for the eight PDI protons, a sharp 
singlet for the four PMDI protons and three sets of signals for the six methine protons, while that of (-)-2NDI-1PDI- $\Delta$ shows (Figure 2 and Figure S3) three sets of signals for the eight PDI protons, two sets of signals for the eight NDI protons and two sets of signals for the six methine protons.

Furthermore, the conformational rigidity of these isosceles triangles could be established by dynamic ${ }^{1} \mathrm{H}$ NMR experiments by probing the rates of rotation of the aromatic subunits around their $\mathrm{C}-\mathrm{N} \bullet \bullet \mathrm{N}-\mathrm{C}$ bond axes. Based on our previous observations, ${ }^{54-55}$ we thought it would be unlikely to surmount the high free energy of activation necessary for the rotation of the bulky NDI and PDI subunits around their $\mathrm{C}-\mathrm{N} \bullet \bullet \mathrm{N}-\mathrm{C}$ bond axes within the triangles on the ${ }^{1} \mathrm{H}$ NMR time scale. In any event, the accidental chemical shifts of the resonances for the heterotopic PMDI protons of (-)-2PMDI-1PDI- $\Delta$, affording a sharp singlet (Figure S1) even at room temperature, restricted our attempts (Figure S6) to probe the rates of rotation of the PMDI subunits with increasing temperature. The downfield shift of the resonances observed for the aromatic protons of (-)-2PMDI-1PDI- $\Delta$ (Figure S6) and (-)-2NDI-1PDI- $\Delta$ (Figure S7) can be attributed to the weakening of the $\pi$-stacking intermolecular interactions between the aromatic subunits upon increasing the temperature.

Quantum mechanical (QM) calculations. In order to investigate the conformational rigidity of the aromatic subunits within the isosceles triangles, we carried out quantum mechanical calculations (Figures S8 and S9) to map the potential energy surface as a function of the rotational barriers of the dihedral angle $\angle(\mathrm{H}-\mathrm{C}-\mathrm{N}-\mathrm{C})$ for all the aromatic PDI, NDI and PMDI subunits (Figure S9) present in all the compounds. In the case of Ref-PDI, the activation barrier to the rotation of PDI is only $6.85 \mathrm{kcal} \mathrm{mol}^{-1}$. This barrier increases dramatically to 25.4 and $25.1 \mathrm{kcal}$ $\mathrm{mol}^{-1}$ for (-)-2PMDI-1PDI- $\Delta$ and (-)-2NDI-1PDI- $\Delta$, respectively. This observation suggests that their rigid cyclic structures restrict the free intramolecular rotation of the PDI components. 
After investigating the rigidity of the isosceles triangles, we conducted thermogravimetric analyses under a nitrogen atmosphere in order to determine their thermal stabilities. Although an initial mass loss of up to $4 \%$ below $250{ }^{\circ} \mathrm{C}$ was observed (Figure S12) for (-)-2PMDI-1PDI-A and (-)-2NDI-1PDI- $\Delta$, when compared to that of Ref-PDI, the triangles exhibited high thermal stability up to $410{ }^{\circ} \mathrm{C}$ whereupon they began to decompose. Moreover, variable-temperature powder X-ray diffraction (VT-PXRD) studies were performed on the as-synthesized powder samples of the isosceles triangles to obtain insights into their thermal stability, crystallinity and phase purity. The PXRD patterns of (-)-2PMDI-1PDI- $\Delta$ at room temperature indicate (Figure S10) that it is semi-crystalline, while the sharpening of peaks with the rise in temperature up to $443 \mathrm{~K}$ suggests its increased crystallinity. The original degree of crystallinity is, however, obtained upon subsequent cooling to room temperature. On the other hand, PXRD patterns of (-)-2NDI1PDI- $\Delta$ at room temperature reveal (Figure S11) its crystalline structure which is, not only thermally stable up to $473 \mathrm{~K}$, but also retains the same crystalline structure upon subsequent cooling to room temperature. In addition to their superior structural and conformational rigidity, the remarkable thermal stability of (-)-2PMDI-1PDI- $\Delta$ and (-)-2NDI-1PDI- $\Delta$ is promising for practical applications in organic electronic devices.

Single-crystal X-ray diffraction (XRD) analyses. Single-crystal X-ray diffraction analyses were carried out in order to gain insights into structural details and packing arrangements of these rigid isosceles triangular macrocycles. Single crystals of (-)-2PMDI-1PDI- $\Delta$ were obtained by slow vapor diffusion of $n$-hexane into a $3 \mathrm{mM}$ solution in 1,2-dichloroethane (DCE) over the course of 3 days, while single crystals of (-)-2NDI-1PDI- $\Delta$ were obtained by slow evaporation of a $6 \mathrm{mM}$ solution in $\mathrm{CHCl}_{3}$ over the course of 7 days. The crystal structures of both (-)-2PMDI-1PDI- $\Delta$ and (-)-2NDI-1PDI- $\Delta$ reveal (Figure $3 \mathrm{a}$ and $\mathrm{b}$ ) the strained rigid geometries of the isosceles 
triangular hollow prisms which are characterized by similar vertex angles (Figure $3 \mathrm{c}$ and e) of $\sim 88^{\circ}-$ a value which is much greater than the $60^{\circ}$ observed for the equilateral triangle (-)-3NDI$\Delta^{50-51,54}$ - as a result of the stretching of the long and curved PDI subunits. On account of the fact that the PMDI units $(9.6 \AA)$ are slightly shorter than the NDI ones $(10.0 \AA)$, the base-to-apex distance $(8.8 \AA)$ of (-)-2PMDI-1PDI- $\Delta$ is shorter than that $(9.5 \AA)$ of (-)-2NDI-1PDI- $\Delta$. The angle $\left(102^{\circ}\right)$ between the PDI and the triangular planes in (-)-2PMDI-1PDI- $\Delta$ (Figure 3d) is larger than that $\left(86^{\circ}\right)$ of $(-)-2$ NDI-1PDI- $\Delta$ (Figure $\left.3 \mathrm{f}\right)$ as a result of the less bulky PMDI when compared with the NDI units. In addition, the PDI plane of (-)-2PMDI-1PDI- $\Delta$ is more bent than the PDI plane of (-)-2NDI-1PDI- $\Delta$. These observations indicate that the triangular structure of (-)2PMDI-1PDI- $\Delta$ is relatively more strained than that of (-)-2NDI-1PDI- $\Delta$. In both crystal superstructures, every two (-)-2PMDI-1PDI- $\Delta$ and every two (-)-2NDI-1PDI- $\Delta$ form (Figure 3cf) nearly face-to-face $\pi-\pi$ dimers by means of $\pi-\pi$ stacking interactions ( $\sim 3.4 \AA)$ between nearly parallel PDI units, respectively. Although both PDI units in the $\pi-\pi$ dimer of (-)-2PMDI-1PDI- $\Delta$ are almost parallel, a longitudinal offset of $0.6 \AA$ and a transverse offset of $1.3 \AA$ are present (Figure $3 \mathrm{c}$ and $\mathrm{d}$ and see supplementary movies of the $\pi-\pi$ dimers) between the two overlaying PDI units. By contrast, in the $\pi-\pi$ dimer of (-)-2NDI-1PDI- $\Delta$, the longitudinal (4.0 $\AA)$ and transverse $(2.0$ $\AA$ ) offsets of two PDI units are much greater (Figure $3 \mathrm{e}$ and f), an observation which indicates that the $\pi-\pi$ stacking interactions between PDI units of (-)-2PMDI-1PDI- $\Delta$ are more efficient than those in (-)-2NDI-1PDI- $\Delta$. This result can be ascribed to the fact that the more bent PDI units of (-)-2PMDI-1PDI- $\Delta$ results in them exposing larger $\pi$-surfaces for $\pi-\pi$ stacking while at the same time weakening the steric barriers from the cyclohexano groups and thus favor the overlap of the larger areas provided by the two PDI units between adjacent (-)-2PMDI-1PDI- $\Delta$. In the extended superstructures, every four $\pi-\pi$ dimers of (-)-2PMDI-1PDI- $\Delta$ and (-)-2NDI-1PDI- $\Delta$ pack in 
different orientations in the presence of solvent molecules within a unit cell. Notably, no significant noncovalent bonding interactions were found; namely, $\pi-\pi$ stacking and $[\mathrm{C}-\mathrm{H} \cdots \mathrm{O}]$ interactions between PMDI/PMDI, PMDI/PDI, NDI/NDI, or NDI/PDI pairs which are very commonly observed previously in the cases of (-)-3NDI- $\Delta,{ }^{50-51,54}(-)-1$ PMDI-2NDI- $\Delta$, and (-)2PMDI-1NDI- $\Delta$ triangles $^{55}$. In addition, the X-ray structures reveal that the nanoporous cavities of both isosceles triangles are indeed encapsulated by the solvent molecules as evidenced by (-)2PMDI-1PDI- $\Delta$ forming (Figure S14) a 2:1 host-guest complex with $n$-hexane as a result of multiple $[\mathrm{C}-\mathrm{H} \cdots \pi]$ interactions, while $\mathrm{CHCl}_{3}$ molecules are bound (Figure S15) to the cavities of (-)-2NDI-1PDI- $\Delta$ stabilized by multiple $[\mathrm{Cl} \cdots \pi]$ interactions $(\sim 3.4 \AA)$ with the $\pi$-surfaces of NDI and PDI subunits.

On the other hand, Hirshfeld surface analyses ${ }^{64}$ performed on the structures of the two isosceles triangles confirmed (Figure S18) that the reciprocal $\left[\pi^{\cdots} \pi\right]$ interactions, which contribute about 12.2 and $12.1 \%$ to the Hirshfeld surfaces of (-)-2PMDI-1PDI- $\Delta$ and (-)-2NDI-1PDI- $\Delta$, respectively, are the most significant interactions between the PDI units in the case of both $\pi-\pi$ dimers. In order to assess the robustness of such discrete nearly cofacial $\pi \cdots \pi$ dimer packing motifs, several attempts have been made to crystallize these triangles from a range of different solvents. Although single crystals suitable for XRD analysis could not be obtained in most cases, (-)2PMDI-1PDI- $\Delta$ did crystallize from the slow vapor diffusion of $n$-hexane into a $3 \mathrm{mM}$ solution of (-)-2PMDI-1PDI- $\Delta$ in $\mathrm{CHCl}_{3}$ over the course of several days. Single-crystal XRD analysis reveals (Figure S16) that (-)-2PMDI-1PDI- $\Delta$ also forms discrete nearly cofacial PDI-PDI $\pi$ dimers which exhibit identical packing arrangements and the unit cell parameters to those observed for the DCE $/ n$-hexane system. See Figure 3 and details of the crystallographic characterization in the Supporting Information. Also, in order to understand the role of solvents on the formation of 
such discrete PDI-PDI $\pi$-dimers in the solid state, solvent-free single crystals of (-)-2PMDI1PDI- $\Delta$, suitable for XRD analysis, were obtained by air drying the single crystals grown by slow vapor diffusion of $n$-hexane in $\mathrm{CHCl}_{3}$ solution. It was observed (Figure S17) that the discrete PDIPDI $\pi$-dimers remained intact, with no change in the unit cell parameters before and after the evaporation of $\mathrm{CHCl}_{3}$ from the single crystals. Based on all of these observations, it can be concluded that the geometries of the triangular hollow prisms of (-)-2PMDI-1PDI- $\Delta$ and (-)2NDI-1PDI- $\Delta$ facilitate specifically the formation only of the discrete nearly cofacial $\pi-\pi$ dimers involving two PDI units by preventing the PDI-PDI $\pi$-dimers from further long-range $\pi-\pi$ stacking and aggregation, which otherwise occurs ${ }^{65}$ very easily in most of the PDI derivatives. Therefore, we anticipated that the presence of the geometrically protected rigid discrete PDI-PDI $\pi$-dimers of the triangles may be reflected in their solid-state photoluminescence properties.

Photophysical studies in solution. Motivated by the variation in the structural properties and the unusual packing arrangements of the rigid isosceles triangles in the solid state, as evidenced by single-crystal XRD (Figure 3), we set out to investigate (i) the optical properties of all three compounds by steady-state UV/Vis absorption and fluorescence spectroscopies (Table 1 and Figure 4), and (ii) the chiroptical behavior of the isosceles triangles by CD spectroscopy in solution. The absorption spectra (Figure $4 \mathrm{a}, \mathrm{b}$ and c) of all three compounds recorded in $\mathrm{CH}_{2} \mathrm{Cl}_{2}$ displayed three well-defined vibronic bands with maxima between 456-459, 487-490 and 524-527 nm, corresponding to the characteristic $\mathrm{S}_{1} \leftarrow \mathrm{S}_{0}$ electronic transition of the PDI derivatives. This observation suggests that the imide substituents of PDI subunits present in all three compounds have negligible impact on their electronic transitions. Additionally, the absorption spectra (Figure 4c) of (-)-2NDI-1PDI- $\Delta$ displayed two vibronic progressions, centered on 360 and $380 \mathrm{~nm}$, corresponding to the characteristic $\mathrm{S}_{1} \leftarrow \mathrm{S}_{0}$ electronic transition of the NDI subunits. The molar 
extinction coefficients of both the isosceles triangles are smaller (Table 1) than that of Ref-PDI. Consequently, the CD spectra of both chiral isosceles triangles displayed (Figure S13) prominent negative exciton Cotton effects in the 225-250, 350-400 and 450-550 nm regions, corresponding to the electronic transitions of the PMDI, NDI and PDI subunits, respectively, where the sign of the peaks is consistent with the absolute $(R R R R R R)$-configuration of the triangles. The fluorescence spectra (Figure $4 \mathrm{a}, \mathrm{b}$ and $\mathrm{c}$ ) of all three compounds showed similar monomeric emission bands with mirror-image vibronic patterns to their absorption spectra. The fluorescence quantum yield measured in $\mathrm{CH}_{2} \mathrm{Cl}_{2}$ relative to that of Ref-PDI $\left(\Phi_{\mathrm{f}}=100 \%\right)^{44}$ is almost unity $\left(\Phi_{\mathrm{f}}\right.$ $=100 \%)$ for (-)-2PMDI-1PDI- $\Delta$, while that for (-)-2NDI-1PDI- $\Delta$ is only slightly lower $\left(\Phi_{\mathrm{f}}=\right.$ $88 \%$ ). Similarly, all three compounds also exhibited excellent fluorescence quantum yields $\left(\Phi_{\mathrm{f}} \sim\right.$ 90-100\%) in other organic solvents, such as $\mathrm{MeCN}$ and PhMe, except the partially quenched fluorescence of (-)-2NDI-1PDI- $\Delta\left(\Phi_{\mathrm{f}, \mathrm{PhMe}}=63 \%\right)$ in PhMe which can be attributed to aggregation-caused quenching.

The excitation spectra (Figure 4a, b and c) of all three compounds match well with their absorption spectra, suggesting that the emission arises from only one excited species. The excitation spectrum (Figure 4c) of (-)-2NDI-1PDI- $\Delta$ exhibited, however, additional peaks in the region 350-390 $\mathrm{nm}$ which match perfectly with the vibronic progressions corresponding to the electronic transition localized on the NDI subunits in its absorption spectrum. This observation suggests that there is an efficient intramolecular energy transfer from the lowest excited singlet state of NDI to that of the PDI subunit within the isosceles triangle (-)-2NDI-1PDI- $\Delta$ upon photoexcitation of the NDI subunits (Figure S19). Furthermore, the time-resolved fluorescence spectra (Figure 3d) of all three compounds in $\mathrm{CH}_{2} \mathrm{Cl}_{2}$ displayed mono-exponential decay curves with similar lifetimes $<\tau_{\mathrm{em}}>$ of 4.0, 4.5 and $5.0 \mathrm{~ns}$ for Ref-PDI, (-)-2PMDI-1PDI- $\Delta$ and (-)- 
2NDI-1PDI- $\Delta$, respectively. Additional insights into the excited state dynamics of all three compounds were obtained (Figures S20-S22) using femtosecond transient absorption spectroscopy (fsTA). An excitation wavelength of $493 \mathrm{~nm}$ was chosen, which is resonant with the $0 \leftarrow 1$ vibronic band for all three samples. For Ref-PDI, excited-state ${ }^{1 *} \mathrm{PDI}$ is formed (Figure S20a) within the instrument response time, as characterized by positive bands around 350, 700 and $900 \mathrm{~nm}$, which can be assigned to the excited-state absorption (ESA). Meanwhile, negative bands, which are observed at 456, 486, 525, 575 and $615 \mathrm{~nm}$, can be ascribed to the ground state bleach (GSB) and stimulated emission (SE) based on the steady-state measurements. In the subsequent 7 ns, all transient features decay back to the ground state simultaneously. Global fits to the fsTA data with a species-associated model gave two time constants values of $124 \pm 2$ ps and $3.50 \pm 0.03 \mathrm{~ns}$, which can be assigned (Figure S20) to conformational relaxation and decay of the first singlet excited state $\mathrm{S}_{1}$ to the ground state $\mathrm{S}_{0}$, respectively. Interestingly, the two isosceles triangles exhibited (Figures S21 and S22) similar excited state dynamics to those of Ref-PDI. The observed conformational relaxation timescales are $181 \pm 6$ and $58 \pm 2 \mathrm{ps}$, and the singlet lifetimes are 3.92 \pm 0.05 and $3.44 \pm 0.04 \mathrm{~ns}$, for (-)-2PMDI-1PDI- $\Delta$ (Figure S21) and (-)-2NDI-1PDI- $\Delta$ (Figure S22), respectively. All of these observations reflect that the functionalization of the PDI subunit with chiral cyclic $N$-substituents does not have any significant effect on the excited state dynamics of the PDIs in solution.

Photophysical studies in the solid state. In contrast with the optical properties observed in solution, the optical properties of all three compounds in the solid state differ (Table 2) from one another. The UV/Vis absorption spectra (Figure 5a-c) of thin films and diffuse reflectance spectra (Figure S23) of powder samples were recorded for all three compounds. The UV/Vis absorption 
spectrum of Ref-PDI displays (Figure 5a) typical signatures of excitonically coupled aromatic subunits which can be attributed to strong $\pi-\pi$ overlap between the closely arranged neighboring molecules. The UV/Vis absorption spectra of (-)-2PMDI-1PDI- $\Delta$ and (-)-2NDI-1PDI- $\Delta$, however, show (Figure 5b and c) well-resolved vibronic patterns similar to those found in solution, indicative of only weak interactions between the aromatic subunits in the solid state. It is noteworthy that the intermolecular $\pi$ - $\pi$ stacking between the neighboring molecules in the case of Ref-PDI is consecutive and uninterrupted in the solid state, ${ }^{66}$ while the $\pi$ - $\pi$ stacking in the case of both the isosceles triangles is suppressed, as a result of the formation of discrete $\pi$-dimers lacking any additional long-range noncovalent bonding interactions, on account of their unique rigid triangular geometries as evidenced (Figure 3) by their solid-state (super)structures.

Unlike the excellent fluorescence properties observed in solution, the photoluminescence (PL) of all three compounds led (Table 2 and Figure 5) to aggregation-caused quenching in the solid state. The PL spectra (Figure 5a, b and c) of all three compounds displayed weak, red-shifted excimer emission bands at 652, 670 and $602 \mathrm{~nm}$ for Ref-PDI, (-)-2PMDI-1PDI- $\Delta$ and (-)-2NDI1PDI- $\Delta$, respectively. This variation in the emission maxima of all three compounds reflects the difference in electronic coupling between the two PDIs in the solid-state structure that leads to excimer state stabilization. The greater $\pi-\pi$ overlap of the PDI molecules observed in the crystal structure of (-)-2PMDI-1PDI- $\Delta$ relative to that of (-)-2NDI-1PDI- $\Delta$ is consistent with the larger red shift of the excimer emission from (-)-2PMDI-1PDI- $\Delta$. As a consequence of long, continuous $\pi-\pi$ stacking between neighboring Ref-PDI molecules, negligible photoluminescence quantum yields are observed for Ref-PDI, in both powder form $\left(\Phi_{\text {powder }}=0.1 \%\right)$ and thin film $\left(\Phi_{\text {film }}=0.2 \%\right)$. In contrast, the photoluminescence behavior is somewhat improved (Figure S24) for the isosceles triangles but the quantum yields are still low in the solid state for (-)-2PMDI-1PDI- $\Delta$ ( $\Phi_{\text {powder }}=$ 
$3 \%$ and $\left.\Phi_{\text {film }}=2 \%\right)$ and (-)-2NDI-1PDI- $\Delta\left(\Phi_{\text {powder }}=4 \%\right.$ and $\left.\Phi_{\text {film }}=2 \%\right)$. The photographs (Figure 5e, f and $\mathrm{g}$ ) of all three compounds in powder form, as well as drop-casting films coated on a glass substrate, taken under $365 \mathrm{~nm}$ light irradiation reveal that (-)-2PMDI-1PDI- $\Delta$ and (-)-2NDI1PDI- $\Delta$ exhibit red and orange emissions, respectively, while the emission of Ref-PDI is quenched almost completely. Although the X-ray diffraction patterns obtained using the powder (Figures S10 and S11) and thin film (Figure S25) samples of both the isosceles triangles at room temperature may suggest the peaks corresponding to intermolecular $\pi-\pi$ stacking within the range of 3.2 to $3.7 \AA$ between the aromatic units, this level of information was not sufficient to determine and compare specific molecular packing arrangements in the bulk samples with those observed (Figure 3) by single-crystal XRD. It should be noted that the single-crystal XRD analyses reveal the random orientation of the discrete PDI-PDI $\pi$-dimers throughout the (super)structures in the case of both isosceles triangles and thus, we suppose it may be challenging to experimentally observe the XRD peaks characteristic of packing arrangements associated with $\pi$ - $\pi$ stacking in the powder or thin film samples. Based on the presence and the robustness of the nearly cofacial PDI $\pi-\pi$ dimer packing motifs of the isosceles triangles in the presence of different solvents (Figure 3 and Figures S14-S16) and even under solvent-free conditions (Figure S17), we believe that the improvement in the photoluminescence quantum yields of (-)-2PMDI-1PDI- $\Delta$ and (-)-2NDI1PDI- - — by about 10 to 40 times that of Ref-PDI — in the solid state is, however, a consequence of suppressing the global $\pi$ - $\pi$ stacking interactions defined by their unique structurally rigid triangular geometries.

The solid-state excitation spectra of all three compounds were recorded (Figure 5d) in powder form. Unlike the excitation spectra of Ref-PDI and (-)-2PMDI-1PDI- $\Delta$, the presence of NDI vibronic patterns in the region 340-390 nm for (-)-2NDI-1PDI- $\Delta$ suggests that efficient 
energy transfer occurs between the excited singlet state of NDI and that of the PDI subunit upon photoexcitation of the NDI subunits, even in the solid state. The excimer fluorescence of Ref-PDI and (-)-2NDI-1PDI- $\Delta$ in the solid state display (Figure S26a and S26c) multi-exponential decays with average lifetimes of $\left\langle\tau_{\mathrm{em}}>=1.5\right.$ and $3.0 \mathrm{~ns}$, respectively, while the corresponding excimer fluorescence decay of (-)-2PMDI-1PDI- $\Delta$ exhibits (Figure S26b) a longer multi-exponential decay with an average lifetime of $<\tau_{\mathrm{em}}>=13.4 \mathrm{~ns}$, which again is consistent with the more highly constrained cofacial geometry of the PDI-PDI $\pi$-dimers in (-)-2PMDI-1PDI- $\Delta$.

Moreover, fsTA was likewise applied to film samples to interrogate the excited state dynamics. While the poor solubility of Ref-PDI and (-)-2NDI-1PDI- $\Delta$ in $\mathrm{CH}_{2} \mathrm{Cl}_{2}$ prevented our attempts to prepare their homogeneous thin film samples, a suitable drop-casting film sample of (-)-2PMDI-1PDI- $\Delta$ was prepared. It is evident that the spectra at the early time delays correspond to the $\mathrm{S}_{1}$ state (Figure 6a). Significantly, after about $3 \mathrm{ps}$, the peaks in the $600-800 \mathrm{~nm}$ ESA region are flattened, which is a signature ${ }^{67-68}$ of excimer formation. All transient signals then decay back to $\mathrm{S}_{0}$ in $7 \mathrm{~ns}$. Global fits to the fsTA data with a species-associated model gave (Figure $6 \mathrm{~b}$ ) three time constant values of $0.8 \pm 0.3,16.2 \pm 0.3$ and $382 \pm 8$ ps, respectively. Figures $6 \mathrm{c}$ and $6 \mathrm{~d}$ illustrate the corresponding multiple-wavelength fits and populations of kinetic states of the species, respectively. The first timescale can be readily attributed to excimer formation from the PDI $S_{1}$ state, whereas the latter two timescales correspond to excimer decay back to $S_{0}$.

Frontier molecular orbitals of isosceles triangles. In order to obtain deeper insight into the electronic properties of these fluorescent isosceles triangles, we performed density functional theory (DFT) quantum mechanics (QM) calculations at the M06-2X level of theory using the 6$311 \mathrm{G}(\mathrm{d}, \mathrm{p})$ basis set and including Poisson Boltzmann Continuum Solvation with $\mathrm{CH}_{2} \mathrm{Cl}_{2}$ as the solvent. All the calculations (Figure 7) include post-stage D3 van der Waals corrections. ${ }^{69-70}$ Our 
calculations on (-)-2PMDI-1PDI- $\Delta$ reveal that the HOMO-1 and HOMO-2 are primarily located (Figure $7 \mathrm{a}$ and $\mathrm{b}$ ) at the diimide-based junctions of the cyclohexano linkers and the PDI subunit, while the LUMO and HOMO are located only on the PDI subunit (Figure 7c and d). In the case of (-)-2NDI-1PDI- $\Delta$, both HOMO-1 and HOMO-2 are located (Figure 7e and f) on the two NDI subunits. On the other hand, the HOMO of (-)-2NDI-1PDI- $\Delta$ is located on the PDI subunit, while the LUMO is located mostly on the PDI subunit but with partial delocalization onto the two NDI subunits (Figure $7 \mathrm{~g}$ and $\mathrm{h}$ ). The electronic transitions for both the isosceles triangles were calculated using a tight-binding approximation of time-dependent density functional theory (TDDFTB). Additionally, the simulated UV/Vis spectra (Figure S27) show that the $\mathrm{S}_{1} \leftarrow \mathrm{S}_{0}$ electronic transitions around $560 \mathrm{~nm}$ correspond to transitions from the HOMO to LUMO located on the PDI subunits. Additionally, the graphical representations obtained from our calculations (Figure 7i and j) show that the PDI planes of both isosceles triangles are significantly curved when compared to the fully relaxed PDI component of Ref-PDI, presumably as a consequence of rigidity and strain $\left(\Delta E \sim 11.8 \mathrm{kcal} \mathrm{mol}^{-1}\right)$ associated with their cyclic triangular geometries, an observation which is consistent (Figure 3) with the single-crystal XRD data.

Cyclic voltammetry (CV). In order to evaluate the potential applicability of the isosceles triangles in optoelectronic devices, we investigated the electrochemical characteristics of (-)-2PMDI1PDI- $\Delta$ and (-)-2NDI-1PDI- $\Delta$ by CV (Figure 8 and Figure S28), and compared these results with those of the monomeric reference compounds, Ref-PMDI, Ref-NDI and Ref-PDI. The CVs of the monomeric reference compounds in $\mathrm{CH}_{2} \mathrm{Cl}_{2}$ exhibited two distinct reversible one-electron redox waves with half-wave potentials at (i) -925 and $-1527 \mathrm{mV} v s \mathrm{Ag} / \mathrm{AgCl}$ for Ref-PMDI, (ii) -709 and $-1131 \mathrm{mV}$ for Ref-NDI, and (iii) -605 and $-794 \mathrm{mV}$ for Ref-PDI, corresponding to the formation of radical anions and dianions, respectively. Strikingly, the CV of (-)-2PMDI-1PDI- $\Delta$ 
displayed (Figure 8) four distinct redox processes involving a total of six-electrons — namely, (i) two sequential and distinct reversible one-electron waves at -566 and $-832 \mathrm{mV}$, corresponding to the formation of $\mathbf{P D I}^{--}$and $\mathbf{P D I}^{2-}$, respectively, (ii) a reversible two-electron wave at $-1008 \mathrm{mV}$, corresponding to the formation of two $\mathbf{P M D I}^{{ }^{-}}$radical anions and (iii) finally, a quasi-reversible two-electron process at $-1764 \mathrm{mV}$, corresponding to the formation of two PMDI ${ }^{2-}$ dianions. In addition, the CV of (-)-2NDI-1PDI- $\Delta$ exhibited (Figure 8) five distinct redox processes involving six electrons in total - namely, (i) a reversible one-electron wave at $-572 \mathrm{mV}$, corresponding to the formation of a singly reduced $\mathbf{P D I}{ }^{--}$radical anion, (ii) a reversible two-electron wave at -749 $\mathrm{mV}$, corresponding to the formation of two singly reduced NDI $^{-}$species, (iii) a reversible oneelectron wave at $-960 \mathrm{mV}$, corresponding to the formation of $\mathbf{P D I}^{2-}$, and (iv) finally, two reversible one-electron waves at -1331 and $-1479 \mathrm{mV}$, corresponding to the formation of two $\mathrm{NDI}^{2-}$ dianions. The number of electrons involved in each of these redox processes is confirmed by differential pulse voltammetry (DPV, Figure S29). It is worth noting that the unambiguous assignment of the peaks corresponding to the mono radical anionic states of all PDI and NDI subunits present in (-)-2NDI-1PDI- $\Delta$ was confirmed by spectroelectrochemistry (Figure S30). In addition, all PDI-containing compounds can also be oxidized quasi-reversibly (Figure S28) to their corresponding radical cationic states at around $+1700 \mathrm{mV}$. Thus, the availability of multiple easily accessible reversible redox states for both isosceles triangles demonstrates their potential applicability as electron accumulation materials.

EPR and ENDOR spectroscopies. Since the CV data (Figure 8) indicated that the monoradical states of PDIs could be accessed much more easily than those of the NDIs or PMDIs, we confirmed this trend by EPR and ENDOR spectroscopies. We also investigated whether the unpaired electron of the PDI subunit is shared among the adjacent PMDI or NDI subunits within the isosceles 
triangles. While the EPR spectrum (Figure S31a) of [(-)-2PMDI-1PDI- $\mathbf{0}]^{--}$is nearly identical to that of [Ref-PDI $]^{--}$with similar spectral widths and number of lines, the EPR spectrum (Figure S31a) of $[(-)-2 N D I-1 P D I-\Delta]^{--}$is narrow compared to that of [Ref-PDI $]^{--}$, corresponding to a decrease in the magnitude of the nuclear hyperfine interactions. These results indicate that the unpaired electron is localized only on the PDI subunits in the case of [(-)-2PMDI-1PDI- $\Delta]^{0^{--}}$but partially shared with the adjacent NDI subunits in the case of [(-)-2NDI-1PDI- $\Delta]^{--}$. Similarly, the ENDOR spectrum (Figure S31b) of [(-)-2PMDI-1PDI- $\Delta]^{--}$has equal isotropic hyperfine coupling constants $\left(a_{\mathrm{H}}\right)$ compared to that of [Ref-PDI $]^{\cdot-}$, once again indicating that the unpaired electron is isolated within the PDI subunits. On the other hand, the ENDOR spectrum (Figure S31b) of $[(-)-2 \text { NDI-1PDI- } \Delta]^{--}$exhibits a decrease in its hyperfine coupling constant by about $15 \%$ compared to those of $[\text { Ref-PDI }]^{--}$and [(-)-2PMDI-1PDI- $\left.\Delta\right]^{\circ-}$, suggesting a small degree of electron sharing with the adjacent NDI subunits on the time scale $\left(>10^{7} \mathrm{~s}^{-1}\right)$ of the electron-nuclear hyperfine interaction. All these observations indicate strongly that (i) the sequential reductions of the PDI subunit of (-)-2PMDI-1PDI- $\Delta$ into its monoradical and the dianionic states can be easily accessed without being interrupted by its cyclic $N$-substituents, and (ii) the unpaired electron in [(-)-2PMDI-1PDI- $\Delta]^{--}$is completely localized on the PDI subunit in a fashion similar to that of monomeric Ref-PDI.

\section{- CONCLUSIONS}

In summary, we have demonstrated the synthesis of two isosceles triangles-namely, (-)-2PMDI1PDI- $\Delta$ and (-)-2NDI-1PDI- $\Delta-$ based on a one of the first of its kind designs, wherein one large and two small planar $\pi$-conjugated aromatic diimides are introduced into rigid chiral cyclic structures, incorporating three $(R R)$-trans-1,2-cyclohexanediamines. The two PDI-based isosceles triangles have rigid geometries with lower symmetries ( $C_{2}$ point groups), relative to those ( $D_{3}$ point 
groups) of the equilateral triangles [(-)-3NDI- $\Delta$ and (-)-3PMDI- $\Delta]$, as evidenced by the expected differences in their ${ }^{1} \mathrm{H}$ and ${ }^{13} \mathrm{C}$ NMR spectra. Their solid-state (super)structures show that geometrically protected PDI fluorophores of the isosceles triangles can only undergo intermolecular PDI-PDI $\pi$-stacking to form dimers because of the absence of any additional longrange noncovalent bonding interactions. Quantum mechanical calculations reveal that both the isosceles triangles consist of (i) conformationally rigid structures as indicated by their high intramolecular rotational barriers and (ii) significantly curved PDI planes relative to the fully relaxed PDI plane of Ref-PDI. This unusual formation and packing arrangement, associated with the molecular rigidity, of the isolated PDI-PDI $\pi$-dimers of the isosceles triangles has a significant influence on their photoluminescence properties in the solid state. The solid-state photoluminescence quantum yields observed for the excimer states of (-)-2PMDI-1PDI- $\Delta$ and (-)-2NDI-1PDI- $\Delta$ are about 10 to 40-fold larger compared with that of Ref-PDI. The fluorescence emission spectra also suggest that efficient intramolecular energy transfer occurs between the adjacent NDI and PDI subunits of (-)-2NDI-1PDI- $\Delta$. Such variations in the photophysical properties observed between the monomeric reference compound and the two isosceles triangles could form a basis for the rational design of highly efficient fluorescent organic materials for applications in optoelectronic and photonic devices. Also, both (-)-2PMDI-1PDI- $\Delta$ and (-)2NDI-1PDI- $\Delta$ are chiral molecules with strong fluorescence emissions, and hence they would be expected to exhibit circularly polarized luminescence (CPL). ${ }^{23}$ Moreover, the electrochemical properties investigated by CV indicate that Ref-PDI can only produce two redox states, while (-)2PMDI-1PDI- $\Delta$ and (-)-2NDI-1PDI- $\Delta$ produce multiple easily accessible redox states, suggesting their potential use as electron accumulation or transport materials. The EPR and 
ENDOR spectra show that the unpaired electron in (-)-2PMDI-1PDI- $\Delta$ is localized on the PDI subunit, while it is partially shared between the NDI and PDI subunits in (-)-2NDI-1PDI- $\Delta$.

Based on this strategy of synthesizing PMDI/NDI/PDI cyclic trimers, it should be possible to prepare higher-order macrocyclic oligomers by introducing various other redox-active functional aromatic diimides, including anthracene diimides (ADIs), coronene diimides (CDIs), terrylene diimides (TDIs) and quaterrylene diimides (QDIs). The general features of this synthetic strategy are to make use of smaller aromatic diimides, such as PMDIs and NDIs, as solubilizers so as to incorporate larger insoluble aromatic diimides, without any bulky substituents, easily into these cyclic systems exhibiting multi-functional structural, optical, electronic and magnetic properties associated with their chirality, rigidity, accessible cavities, through-space electron sharing and several readily available redox states. This approach may hold great promise for the design and synthesis of new active materials, as well as establishing their structure-performance relationships, in organic optoelectronics, energy storage and energy harvesting devices.

\section{- ASSOCIATED CONTENT}

\section{Supporting Information}

Materials and methods, synthetic procedures and characterization, NMR spectroscopic data, PXRD and thin film XRD data, photophysical data, computational details and supportive figures. This material is available free of charge via the Internet at http://pubs.acs.org.

\section{- AUTHOR INFORMATION}

\section{Corresponding Author}

*m-wasielewski@northwestern.edu; stoddart@northwestern.edu

\section{Notes}


The authors declare no competing financial interest.

\section{- ACKNOWLEDGEMENTS}

We would like to thank Yilei Wu for his assistance with spectroscopy experiments, Charlotte L. Stern for performing single-crystal XRD studies and Sumit Kewalramani for performing thin film XRD. This research is a part of the Joint Center of Excellence in Integrated Nano-Systems (JCIN) at King Abdulaziz City for Science and Technology (KACST) and Northwestern University (NU). The authors would like to thank both KACST, NU, Tianjin University and the University of New South Wales for their continued support of this research. This work was supported by the U.S. Department of Energy, Office of Science, Office of Basic Energy Sciences under Award DEFG02-99ER14999 (M.R.W.). The quantum mechanics (QM) calculations used the resources of the Extreme Science and Engineering Discovery Environment (XSEDE) which is supported by National Science Foundation grant number ACI-1053575. 


\section{- REFERENCES}

1. Friend, R.; Gymer, R.; Holmes, A.; Burroughes, J.; Marks, R.; Taliani, C.; Bradley, D.; Dos Santos, D.; Bredas, J.; Lögdlund, M., Electroluminescence in Conjugated Polymers. Nature 1999, 397, 121-128.

2. Grimsdale, A. C.; Leok Chan, K.; Martin, R. E.; Jokisz, P. G.; Holmes, A. B., Synthesis of LightEmitting Conjugated Polymers for Applications in Electroluminescent Devices. Chem. Rev. 2009, 109, 897-1091.

3. Duan, L.; Qiao, J.; Sun, Y.; Qiu, Y., Strategies to Design Bipolar Small Molecules for OLEDs: Donor-Acceptor Structure and Non-Donor-Acceptor Structure. Adv. Mater. 2011, 23, 1137-1144.

4. Zaumseil, J.; Sirringhaus, H., Electron and Ambipolar Transport in Organic Field-Effect Transistors. Chem. Rev. 2007, 107, 1296-1323.

5. Weil, T.; Vosch, T.; Hofkens, J.; Peneva, K.; Müllen, K., The Rylene Colorant Family-Tailored Nanoemitters for Photonics Research and Applications. Angew. Chem., Int. Ed. 2010, 49, 90689093.

6. Dodabalapur, A.; Chandross, E. A.; Berggren, M.; Slusher, R. E., Organic Solid-State Lasers: Past and Future. Science 1997, 277, 1787-1788.

7. Samuel, I. D. W.; Turnbull, G. A., Organic Semiconductor Lasers. Chem. Rev. 2007, 107, 12721295.

8. Thomas, S. W.; Joly, G. D.; Swager, T. M., Chemical Sensors Based on Amplifying Fluorescent Conjugated Polymers. Chem. Rev. 2007, 107, 1339-1386.

9. Birks, J. B., Photophysics of Aromatic Molecules. Wiley: London, 1970.

10. Beddard, G.; Porter, G., Concentration quenching in chlorophyll. Nature 1976, 260, 366-367.

11. Qin, T.; Zhou, G.; Scheiber, H.; Bauer, R. E.; Baumgarten, M.; Anson, C. E.; List, E. J. W.; Müllen, K., Polytriphenylene Dendrimers: A Unique Design for Blue-Light-Emitting Materials. Angew. Chem., Int. Ed. 2008, 47, 8292-8296.

12. Lee, Y.-T.; Chiang, C.-L.; Chen, C.-T., Solid-State Highly Fluorescent Diphenylaminospirobifluorenylfumaronitrile Red Emitters for Non-Doped Organic Light-Emitting Diodes. Chem. Commun. 2008, 217-219.

13. Ramanan, C.; Smeigh, A. L.; Anthony, J. E.; Marks, T. J.; Wasielewski, M. R., Competition between Singlet Fission and Charge Separation in Solution-Processed Blend Films of 6,13Bis(triisopropylsilylethynyl)pentacene with Sterically-Encumbered Perylene-3,4:9,10bis(dicarboximide)s. J. Am. Chem. Soc. 2012, 134, 386-397. 
14. Iida, A.; Yamaguchi, S., Intense Solid-State Blue Emission with a Small Stokes' Shift: $\pi$-Stacking Protection of the Diphenylanthracene Skeleton. Chem. Commun. 2009, 3002-3004.

15. An, B.-K.; Kwon, S.-K.; Jung, S.-D.; Park, S. Y., Enhanced Emission and Its Switching in Fluorescent Organic Nanoparticles. J. Am. Chem. Soc. 2002, 124, 14410-14415.

16. Kaiser, T. E.; Wang, H.; Stepanenko, V.; Würthner, F., Supramolecular Construction of Fluorescent J-Aggregates Based on Hydrogen-Bonded Perylene Dyes. Angew. Chem., Int. Ed. 2007, $46,5541-5544$.

17. Zhao, C.-H.; Wakamiya, A.; Inukai, Y.; Yamaguchi, S., Highly Emissive Organic Solids Containing 2,5-Diboryl-1,4-phenylene Unit. J. Am. Chem. Soc. 2006, 128, 15934-15935.

18. Wakamiya, A.; Mori, K.; Yamaguchi, S., 3-Boryl-2,2'-bithiophene as a Versatile Core Skeleton for Full-Color Highly Emissive Organic Solids. Angew. Chem., Int. Ed. 2007, 46, 4273-4276.

19. Luo, J.; Xie, Z.; Lam, J. W. Y.; Cheng, L.; Chen, H.; Qiu, C.; Kwok, H. S.; Zhan, X.; Liu, Y.; Zhu, D.; Tang, B. Z., Aggregation-Induced Emission of 1-methyl-1,2,3,4,5-pentaphenylsilole. Chem. Commun. 2001, 1740-1741.

20. Yuan, W. Z.; Lu, P.; Chen, S.; Lam, J. W. Y.; Wang, Z.; Liu, Y.; Kwok, H. S.; Ma, Y.; Tang, B. Z., Changing the Behavior of Chromophores from Aggregation-Caused Quenching to Aggregation-Induced Emission: Development of Highly Efficient Light Emitters in the Solid State. Adv. Mater. 2010, 22, 2159-2163.

21. Mei, J.; Leung, N. L. C.; Kwok, R. T. K.; Lam, J. W. Y.; Tang, B. Z., Aggregation-Induced Emission: Together We Shine, United We Soar! Chem. Rev. 2015, 115, 11718-11940.

22. Kumar, J.; Nakashima, T.; Kawai, T., Circularly Polarized Luminescence in Chiral Molecules and Supramolecular Assemblies. J. Phys. Chem. Lett. 2015, 6, 3445-3452.

23. Longhi, G.; Castiglioni, E.; Koshoubu, J.; Mazzeo, G.; Abbate, S., Circularly Polarized Luminescence: A Review of Experimental and Theoretical Aspects. Chirality 2016, 28, 696-707.

24. Shimizu, M.; Hiyama, T., Organic Fluorophores Exhibiting Highly Efficient Photoluminescence in the Solid State. Chem. Asian J. 2010, 5, 1516-1531.

25. Würthner, F., Perylene Bisimide Dyes as Versatile Building Blocks for Functional Supramolecular Architectures. Chem. Commun. 2004, 1564-1579.

26. Würthner, F.; Saha-Möller, C. R.; Fimmel, B.; Ogi, S.; Leowanawat, P.; Schmidt, D., Perylene Bisimide Dye Assemblies as Archetype Functional Supramolecular Materials. Chem. Rev. 2016, $116,962-1052$. 
27. Görl, D.; Zhang, X.; Würthner, F., Molecular Assemblies of Perylene Bisimide Dyes in Water. Angew. Chem., Int. Ed. 2012, 51, 6328-6348.

28. Anthony, J. E.; Facchetti, A.; Heeney, M.; Marder, S. R.; Zhan, X., n-Type Organic Semiconductors in Organic Electronics. Adv. Mater. 2010, 22, 3876-3892.

29. Zhan, X.; Facchetti, A.; Barlow, S.; Marks, T. J.; Ratner, M. A.; Wasielewski, M. R.; Marder, S. R., Rylene and Related Diimides for Organic Electronics. Adv. Mater. 2011, 23, 268-284.

30. Li, G.; Zhao, Y.; Li, J.; Cao, J.; Zhu, J.; Sun, X. W.; Zhang, Q., Synthesis, Characterization, Physical Properties, and OLED Application of Single BN-Fused Perylene Diimide. J. Org. Chem. 2015, 80, 196-203.

31. Jones, B. A.; Ahrens, M. J.; Yoon, M.-H.; Facchetti, A.; Marks, T. J.; Wasielewski, M. R., HighMobility Air-Stable n-Type Semiconductors with Processing Versatility: Dicyanoperylene3,4:9,10-bis(dicarboximides). Angew. Chem., Int. Ed. 2004, 43, 6363-6366.

32. Zhan, X.; Tan, Z. a.; Domercq, B.; An, Z.; Zhang, X.; Barlow, S.; Li, Y.; Zhu, D.; Kippelen, B.; Marder, S. R., A High-Mobility Electron-Transport Polymer with Broad Absorption and Its Use in Field-Effect Transistors and All-Polymer Solar Cells. J. Am. Chem. Soc. 2007, 129, 7246-7247.

33. Zhang, X.; Lu, Z.; Ye, L.; Zhan, C.; Hou, J.; Zhang, S.; Jiang, B.; Zhao, Y.; Huang, J.; Zhang, S.; Liu, Y.; Shi, Q.; Liu, Y.; Yao, J., A Potential Perylene Diimide Dimer-Based Acceptor Material for Highly Efficient Solution-Processed Non-Fullerene Organic Solar Cells with 4.03\% Efficiency. Adv. Mater. 2013, 25, 5791-5797.

34. Zhou, E.; Cong, J.; Wei, Q.; Tajima, K.; Yang, C.; Hashimoto, K., All-Polymer Solar Cells from Perylene Diimide Based Copolymers: Material Design and Phase Separation Control. Angew. Chem., Int. Ed. 2011, 50, 2799-2803.

35. Li, C.; Wonneberger, H., Perylene Imides for Organic Photovoltaics: Yesterday, Today, and Tomorrow. Adv. Mater. 2012, 24, 613-636.

36. Schmidt, R.; Oh, J. H.; Sun, Y.-S.; Deppisch, M.; Krause, A.-M.; Radacki, K.; Braunschweig, H.; Könemann, M.; Erk, P.; Bao, Z.; Würthner, F., High-Performance Air-Stable n-Channel Organic Thin Film Transistors Based on Halogenated Perylene Bisimide Semiconductors. J. Am. Chem. Soc. 2009, 131, 6215-6228.

37. Krieg, E.; Weissman, H.; Shirman, E.; Shimoni, E.; Rybtchinski, B., A Recyclable Supramolecular Membrane for Size-Selective Separation of Nanoparticles. Nat Nano 2011, 6, 141-146.

38. Würthner, F., Bay-Substituted Perylene Bisimides: Twisted Fluorophores for Supramolecular Chemistry. Pure Appl. Chem. 2006, 78, 2341-2349. 
39. Kazmaier, P. M.; Hoffmann, R., A Theoretical Study of Crystallochromy. Quantum Interference Effects in the Spectra of Perylene Pigments. J. Am. Chem. Soc. 1994, 116, 9684-9691.

40. Zhao, H.-M.; Pfister, J.; Settels, V.; Renz, M.; Kaupp, M.; Dehm, V. C.; Würthner, F.; Fink, R. F.; Engels, B., Understanding Ground- and Excited-State Properties of Perylene Tetracarboxylic Acid Bisimide Crystals by Means of Quantum Chemical Computations. J. Am. Chem. Soc. 2009, 131, 15660-15668.

41. Safont-Sempere, M. M.; Osswald, P.; Radacki, K.; Würthner, F., Chiral Self-Recognition and Self-Discrimination of Strapped Perylene Bisimides by $\pi$-Stacking Dimerization. Chem. Eur. $J$. 2010, $16,7380-7384$.

42. Safont-Sempere, M. M.; Osswald, P.; Stolte, M.; Grüne, M.; Renz, M.; Kaupp, M.; Radacki, K.; Braunschweig, H.; Würthner, F., Impact of Molecular Flexibility on Binding Strength and SelfSorting of Chiral $\pi$-Surfaces. J. Am. Chem. Soc. 2011, 133, 9580-9591.

43. Jiménez, Á. J.; Lin, M.-J.; Burschka, C.; Becker, J.; Settels, V.; Engels, B.; Wurthner, F., StructureProperty Relationships for 1,7-diphenoxy-perylene Bisimides in Solution and in the Solid State. Chem. Sci. 2014, 5, 608-619.

44. Lin, M.-J.; Jiménez, Á. J.; Burschka, C.; Würthner, F., Bay-Substituted Perylene Bisimide Dye with an Undistorted Planar Scaffold and Outstanding Solid State Fluorescence Properties. Chem. Commun. 2012, 48, 12050-12052.

45. Schlosser, F.; Stepanenko, V.; Würthner, F., Perylene Bisimide Macrocycles and their SelfAssembly on HOPG Surfaces. Chem. Commun. 2010, 46, 8350-8352.

46. Schlosser, F.; Sung, J.; Kim, P.; Kim, D.; Würthner, F., Excitation Energy Migration in Covalently Linked Perylene Bisimide Macrocycles. Chem. Sci. 2012, 3, 2778-2785.

47. Liu, Z.; Nalluri, S. K. M.; Stoddart, J. F., Surveying Macrocyclic Chemistry: from Flexible Crown Ethers to Rigid Cyclophanes. Chem. Soc. Rev. 2017, 46, 2459-2478.

48. Wu, Y.; Frasconi, M.; Gardner, D. M.; McGonigal, P. R.; Schneebeli, S. T.; Wasielewski, M. R.; Stoddart, J. F., Electron Delocalization in a Rigid Cofacial Naphthalene-1,8:4,5-bis(dicarboximide) Dimer. Angew. Chem., Int. Ed. 2014, 53, 9476-9481.

49. Samanta, A.; Liu, Z.; Nalluri, S. K. M.; Zhang, Y.; Schatz, G. C.; Stoddart, J. F., Supramolecular Double-Helix Formation by Diastereoisomeric Conformations of Configurationally Enantiomeric Macrocycles. J. Am. Chem. Soc. 2016, 138, 14469-14480.

50. Schneebeli, S. T.; Frasconi, M.; Liu, Z.; Wu, Y.; Gardner, D. M.; Strutt, N. L.; Cheng, C.; Carmieli, R.; Wasielewski, M. R.; Stoddart, J. F., Electron Sharing and Anion- $\pi$ Recognition in Molecular Triangular Prisms. Angew. Chem., Int. Ed. 2013, 52, 13100-13104. 
51. Liu, Z.; Liu, G.; Wu, Y.; Cao, D.; Sun, J.; Schneebeli, S. T.; Nassar, M. S.; Mirkin, C. A.; Stoddart, J. F., Assembly of Supramolecular Nanotubes from Molecular Triangles and 1,2Dihalohydrocarbons. J. Am. Chem. Soc. 2014, 136, 16651-16660.

52. Wu, Y.; Young, R. M.; Frasconi, M.; Schneebeli, S. T.; Spenst, P.; Gardner, D. M.; Brown, K. E.; Würthner, F.; Stoddart, J. F.; Wasielewski, M. R., Ultrafast Photoinduced Symmetry-Breaking Charge Separation and Electron Sharing in Perylenediimide Molecular Triangles. J. Am. Chem. Soc. 2015, 137, 13236-13239.

53. Chen, D.; Avestro, A.-J.; Chen, Z.; Sun, J.; Wang, S.; Xiao, M.; Erno, Z.; Algaradah, M. M.; Nassar, M. S.; Amine, K.; Meng, Y.; Stoddart, J. F., A Rigid Naphthalenediimide Triangle for Organic Rechargeable Lithium-Ion Batteries. Adv. Mater. 2015, 27, 2907-2912.

54. Liu, Z.; Sun, J.; Zhou, Y.; Zhang, Y.; Wu, Y.; Nalluri, S. K. M.; Wang, Y.; Samanta, A.; Mirkin, C. A.; Schatz, G. C.; Stoddart, J. F., Supramolecular Gelation of Rigid Triangular Macrocycles through Rings of Multiple $\mathrm{C}-\mathrm{H} \cdots \mathrm{O}$ Interactions Acting Cooperatively. J. Org. Chem. 2016, 81, 2581-2588.

55. Nalluri, S. K. M.; Liu, Z.; Wu, Y.; Hermann, K. R.; Samanta, A.; Kim, D. J.; Krzyaniak, M. D.; Wasielewski, M. R.; Stoddart, J. F., Chiral Redox-Active Isosceles Triangles. J. Am. Chem. Soc. 2016, 138, 5968-5977.

56. Wu, Y.; Krzyaniak, M. D.; Stoddart, J. F.; Wasielewski, M. R., Spin Frustration in the Triradical Trianion of a Naphthalenediimide Molecular Triangle. J. Am. Chem. Soc. 2017, 139, 2948-2951.

57. Wu, Y.; Nalluri, S. K. M.; Young, R. M.; Krzyaniak, M. D.; Margulies, E. A.; Stoddart, J. F.; Wasielewski, M. R., Charge and Spin Transport in an Organic Molecular Square. Angew. Chem., Int. Ed. 2015, 54, 11971-11977.

58. Mizuno, A.; Shuku, Y.; Suizu, R.; Matsushita, M. M.; Tsuchiizu, M.; Reta Mañeru, D.; Illas, F.; Robert, V.; Awaga, K., Discovery of the $K_{4}$ Structure Formed by a Triangular $\pi$ Radical Anion. $J$. Am. Chem. Soc. 2015, 137, 7612-7615.

59. Mizuno, A.; Shuku, Y.; Matsushita, M. M.; Tsuchiizu, M.; Hara, Y.; Wada, N.; Shimizu, Y.; Awaga, K., 3D Spin-Liquid State in an Organic Hyperkagome Lattice of Mott Dimers. Phys. Rev. Lett. 2017, 119, 057201.

60. Gawroński, J.; Brzostowska, M.; Gawrońska, K.; Koput, J.; Rychlewska, U.; Skowronek, P.; Nordén, B., Novel Chiral Pyromellitdiimide (1,2,4,5-Benzenetetracarboxydiimide) Dimers and Trimers: Exploring Their Structure, Electronic Transitions, and Exciton Coupling. Chem. Eur. J. 2002, 8, 2484-2494. 
61. Gawroński, J.; Brzostowska, M.; Kacprzak, K.; Kołbon, H.; Skowronek, P., Chirality of Aromatic Bis-Imides from their Circular Dichroism Spectra. Chirality 2000, 12, 263-268.

62. Shukla, D.; Nelson, S. F.; Freeman, D. C.; Rajeswaran, M.; Ahearn, W. G.; Meyer, D. M.; Carey, J. T., Thin-Film Morphology Control in Naphthalene-Diimide-Based Semiconductors: High Mobility n-Type Semiconductor for Organic Thin-Film Transistors. Chem. Mater. 2008, 20, 74867491.

63. Chen, Z. J.; Wang, L. M.; Zou, G.; Zhang, L.; Zhang, G. J.; Cai, X. F.; Teng, M. S., Colorimetric and Ratiometric Fluorescent Chemosensor for Fluoride Ion based on Perylene Diimide Derivatives. Dyes Pigm. 2012, 94, 410-415.

64. Turner, M.; McKinnon, J.; Wolff, S.; Grimwood, D.; Spackman, P.; Jayatilaka, D.; Spackman, M., CrystalExplorer17. University of Western Australia 2017, http://hirshfeldsurface.net.

65. Che, Y.; Huang, H.; Xu, M.; Zhang, C.; Bunes, B. R.; Yang, X.; Zang, L., Interfacial Engineering of Organic Nanofibril Heterojunctions into Highly Photoconductive Materials. J. Am. Chem. Soc. 2011, 133, 1087-1091.

66. Che, Y.; Yang, X.; Balakrishnan, K.; Zuo, J.; Zang, L., Highly Polarized and Self-Waveguided Emission from Single-Crystalline Organic Nanobelts. Chem. Mater. 2009, 21, 2930-2934.

67. Brown, K. E.; Salamant, W. A.; Shoer, L. E.; Young, R. M.; Wasielewski, M. R., Direct Observation of Ultrafast Excimer Formation in Covalent Perylenediimide Dimers Using NearInfrared Transient Absorption Spectroscopy. J. Phys. Chem. Lett. 2014, 5, 2588-2593.

68. Cook, R. E.; Phelan, B. T.; Kamire, R. J.; Majewski, M. B.; Young, R. M.; Wasielewski, M. R., Excimer Formation and Symmetry-Breaking Charge Transfer in Cofacial Perylene Dimers. J. Phys. Chem. A 2017, 121, 1607-1615.

69. Grimme, S.; Antony, J.; Ehrlich, S.; Krieg, H., A Consistent and Accurate Ab Initio Parametrization Of Density Functional Dispersion Correction (DFT-D) for the 94 Elements H-Pu. J. Chem. Phys. 2010, 132, 154104.

70. Goerigk, L.; Grimme, S., A Thorough Benchmark of Density Functional Methods for General Main Group Thermochemistry, Kinetics, and Noncovalent Interactions. Phys. Chem. Chem. Phys. 2011, 13, 6670-6688. 


\section{Captions to Figures}

Figure 1. Synthesis of chiral rigid PDI-based isosceles triangles. Stepwise preparation of the isosceles triangles [(-)-2PMDI-1PDI- $\Delta$ and (-)-2NDI-1PDI- $\Delta]$ from (RR)-trans-1,2cyclohexanediamine, pyromellitic dianhydride (PMDA), naphthalenetetracarboxylic dianhydride (NDA) and perylenetetracarboxylic dianhydride (PDA). The inset shows the structural formulas of the monomeric reference compounds (Ref-PMDI, Ref-NDI and Ref-PDI). The aromatic PMDI, NDI and PDI subunits within the molecular triangles are shown in magenta, blue and green, respectively.

Figure 2. ${ }^{1} \mathrm{H}$ NMR spectral comparison of the molecular triangles. Comparison of the annotated ${ }^{1} \mathrm{H}$ NMR spectra $\left(500 \mathrm{MHz}, \mathrm{CDCl}_{3}, 298 \mathrm{~K}\right)$ of the molecular triangles (-)-3NDI- $\Delta$, (-)2NDI-1PDI- $\Delta,(-)-2 P M D I-1 P D I-\Delta$ and (-)-3PMDI- $\Delta$. The signals corresponding to the aromatic protons of the PDI, NDI and PMDI subunits, and the aliphatic protons of the cyclohexano linkers, are annotated in green, blue, magenta, and black, respectively. The unambiguous assignment of the axial and equatorial protons of the cyclohexano linkers is presented in the Supporting Information.

Figure 3. Single-crystal X-ray structures of (-)-2PMDI-1PDI- $\Delta$ and (-)-2NDI-1PDI- $\Delta$. (a and b) Tubular representations of (a) (-)-2PMDI-1PDI- $\Delta$ and (b) (-)-2NDI-1PDI- $\Delta$. (c-f) Top and side-on views of $\pi-\pi$ stacking dimers of (c and d) (-)-2PMDI-1PDI- $\Delta$ and (e and f) (-)-2NDI1PDI- $\Delta$. g) A view along $c$-axis of the unit cell of (-)-2PMDI-1PDI- $\Delta$. h) A view along $b$-axis of the unit cell of (-)-2NDI-1PDI- $\Delta$. For the sake of clarity, solvent molecules are omitted, and in Figure $\mathbf{g}$ and $\mathbf{h}, \mathrm{H}$-atoms are omitted and four $\pi-\pi$ dimers adopting different orientations are depicted in blue, magenta, red, and green.

Figure 4. Photophysical studies of reference compound and isosceles triangles in solution. Normalized (a-c) steady-state UV/Vis absorption (black solid line), excitation (red dashed line) and fluorescence emission (red solid line) spectra of Ref-PDI (a), (-)-2PMDI-1PDI- $\Delta$ (b) and (-)-2NDI-1PDI- $\Delta$ (c) recorded in $\mathrm{CH}_{2} \mathrm{Cl}_{2}$ at $298 \mathrm{~K}$. d) Fluorescence decay curves of Ref-PDI (black line), (-)-2PMDI-1PDI- $\Delta$ (red line), (-)-2NDI-1PDI- $\Delta$ (blue line). The fluorescence spectra of all three compounds were obtained with the excitation at $493 \mathrm{~nm}$. 
Figure 5. Photophysical studies of reference compound and isosceles triangles in the solid state. Normalized (a-c) UV/Vis absorption spectra (black solid line) of the drop-casting films and photoluminescence (red solid line) spectra of the powder samples of Ref-PDI (a), (-)-2PMDI1PDI- $\Delta$ (b) and (-)-2NDI-1PDI- $\Delta$ (c) recorded at 298 K. (d) Normalized excitation spectra of Ref-PDI, (-)-2PMDI-1PDI- $\Delta$ and (-)-2NDI-1PDI- $\Delta$ recorded in the solid state (powder form) at 298 K. The excitation spectra of Ref-PDI, (-)-2PMDI-1PDI- $\Delta$ and (-)-2NDI-1PDI- $\Delta$ were recorded using the emission wavelengths at 720, 720 and $670 \mathrm{~nm}$, respectively. (e-g) The photoluminescence photographs of the corresponding compounds in powder form (left) as well as drop-casting films coated on a glass substrate (right) under daylight (top) and upon $365 \mathrm{~nm} \mathrm{UV}$ light irradiation (bottom). The photoluminescence spectra of all three compounds were obtained with the excitation at $360 \mathrm{~nm}$.

Figure 6. Femtosecond transient absorption (fsTA) spectra of the drop-cast thin film of (-)2PMDI-1PDI- $\Delta$. a) fsTA spectra and (b) species-associated spectra (SAS) and (c) multiplewavelength fits and (d) populations of kinetic states of the thin film of (-)-2PMDI-1PDI- $\Delta$ upon photoexcitation at $493 \mathrm{~nm}$. Different states used in this spectra are represented by $A: S_{1}$, B: excimer state, C: relaxed excimer state and $0: \mathrm{S}_{0}$.

Figure 7. Quantum mechanical calculations on the molecular orbitals of isosceles triangles. (a-d) Graphical representations from HOMO-2 to LUMO of (-)-2PMDI-1PDI- $\Delta$; (e-h) graphical representations from HOMO-2 to LUMO of (-)-2NDI-1PDI- $\Delta$ from DFT calculations at the M06-2X level of theory using 6-311G(d,p) basis sets. (i and $\mathbf{j}$ ) Graphical representations showing the curved PDI plane in (-)-2PMDI-1PDI- $\Delta$ (in yellow) and (-)-2NDI-1PDI- $\Delta$ (in green) in comparison with the fully relaxed PDI component (in blue). The molecular orbital energies are referenced to the molecular orbital energy of HOMO. All the energy units are shown in $\mathrm{kcal} \mathrm{mol}^{-1}$.

Figure 8. Cyclic Voltammetry of isosceles triangles and reference compounds. Cyclic Voltammograms (0.2 mM in $\left.\mathrm{CH}_{2} \mathrm{Cl}_{2}, 100 \mathrm{mM} \mathrm{TBAPF}_{6}, 50 \mathrm{mVs}^{-1}, 298 \mathrm{~K}\right)$ of Ref-NDI, (-)-2NDI1PDI- $\Delta$, (-)-2PMDI-1PDI- $\Delta$, Ref-PDI and Ref-PMDI. Half-wave peak potentials $\left(E_{1 / 2}\right)$ are shown in $\mathrm{mV}$. The oxidation of all PDI-containing compounds is shown in ESI. 

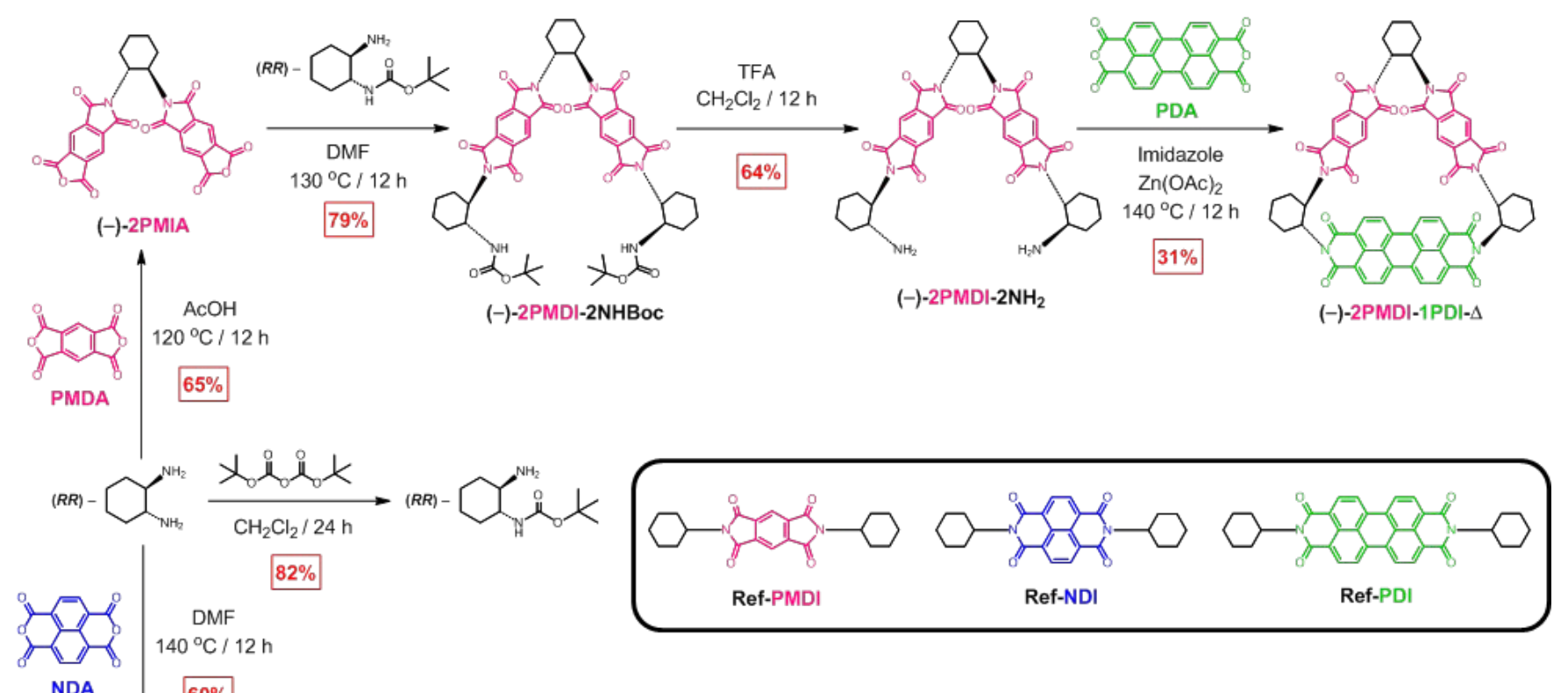
$140^{\circ} \mathrm{C} / 12$

NDA $60 \%$
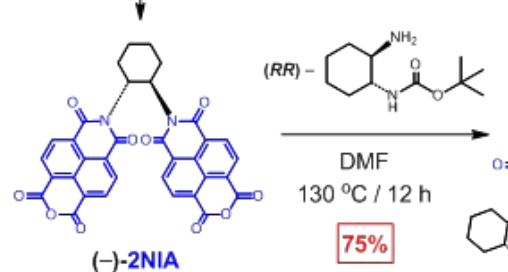

$75 \%$

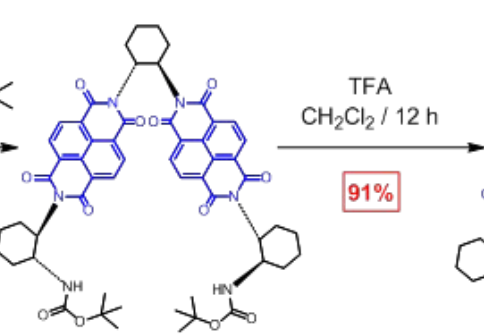

(-)-2NDI-2NHBoc

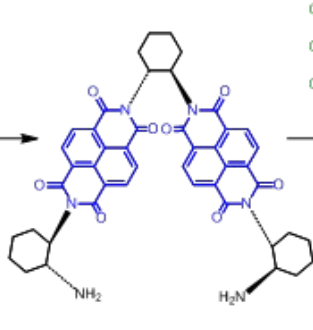

$(-)-2 \mathrm{NDI}^{-2} \mathrm{NH}_{2}$

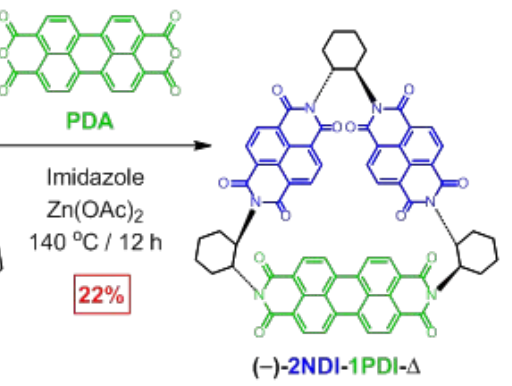

(-)-2NDI-1 PDI-A

Figure 1 
a)

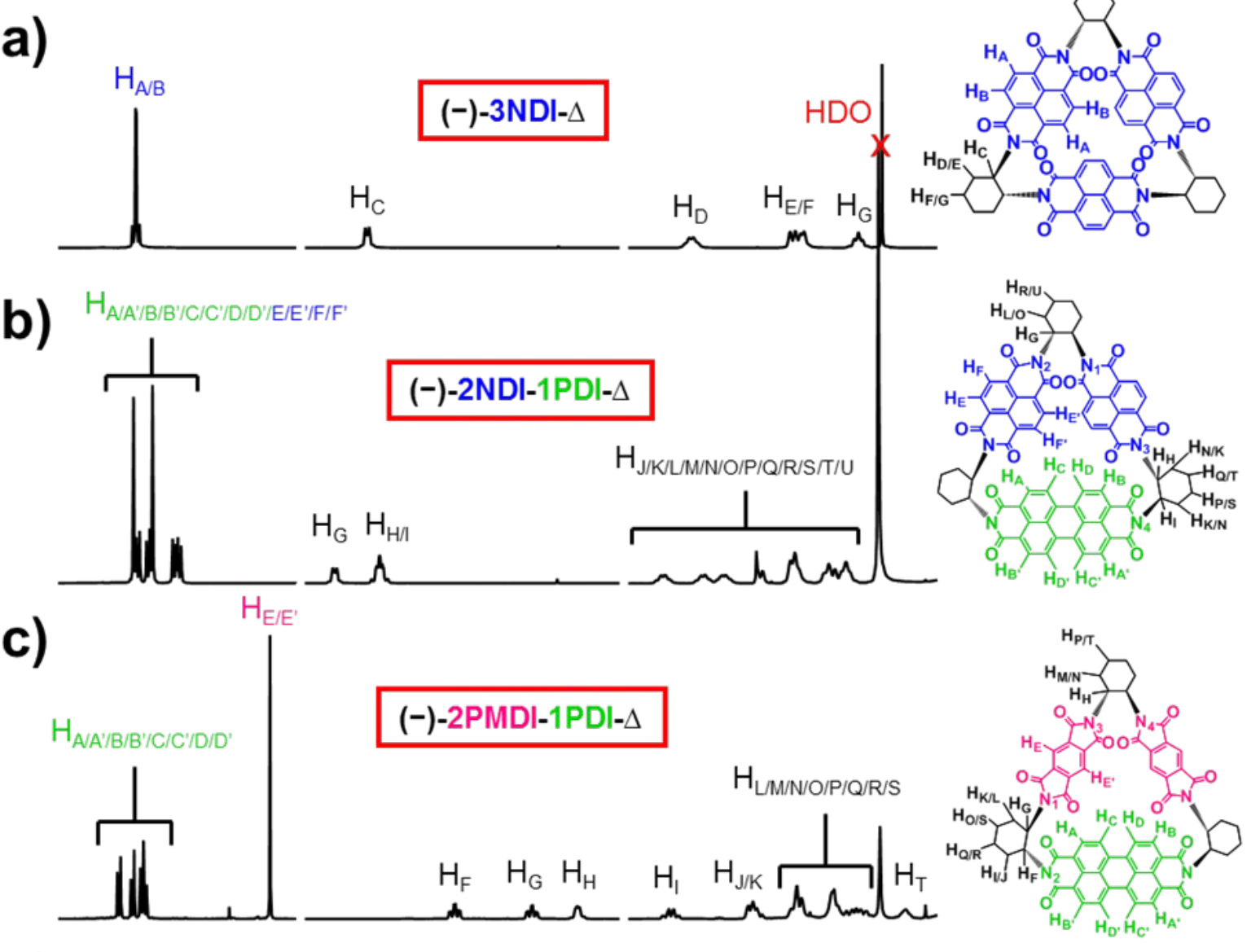

d)

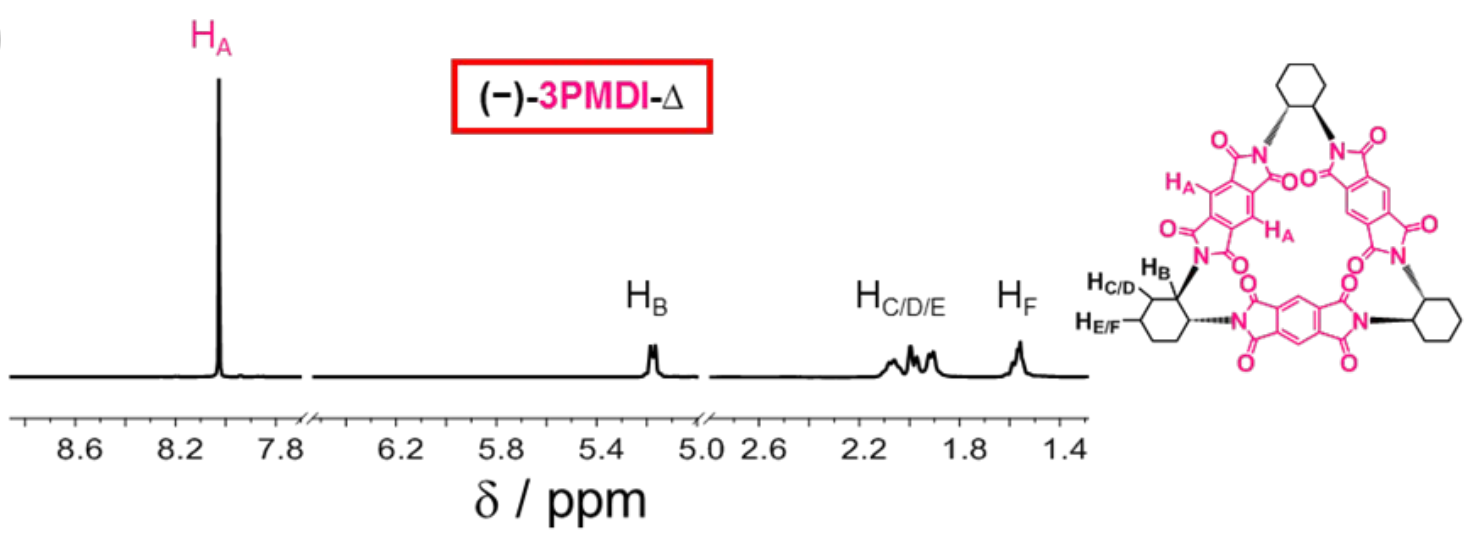

Figure 2 

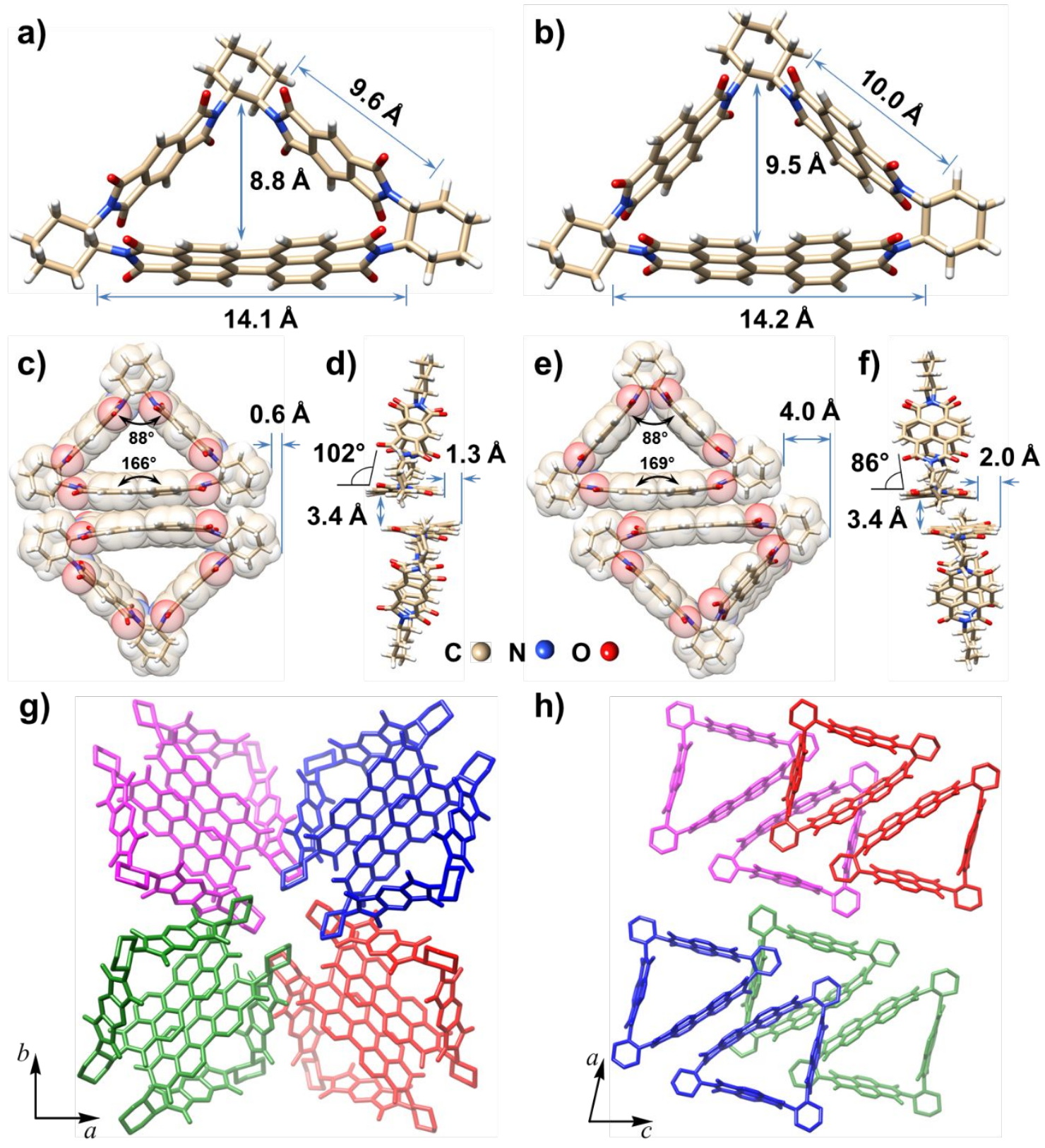

h)

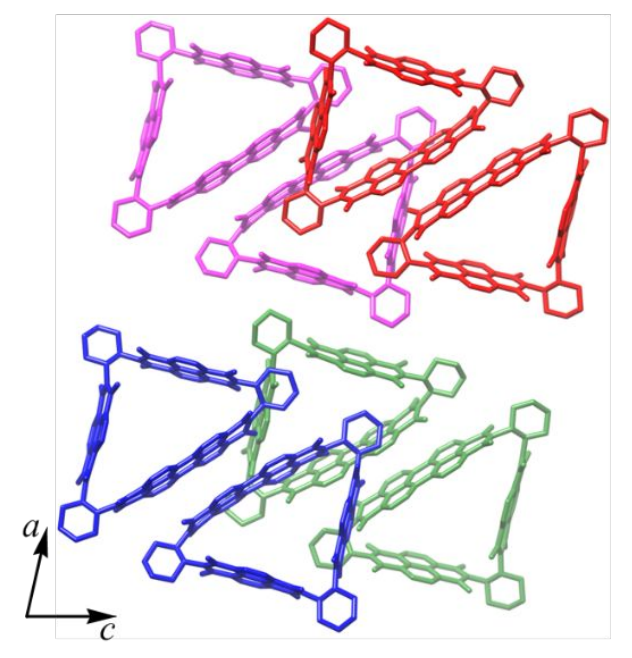

Figure 3 

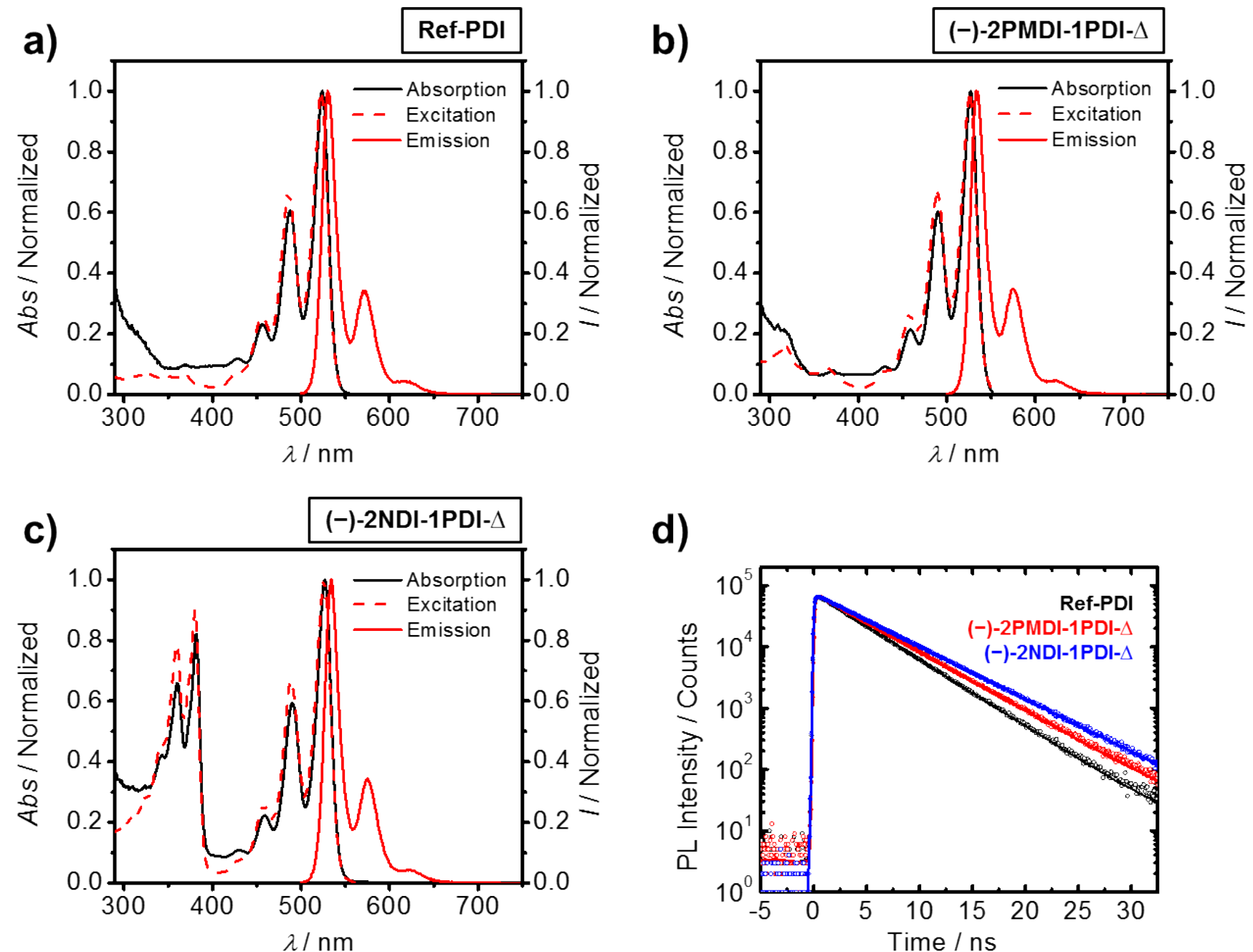

Figure 4 


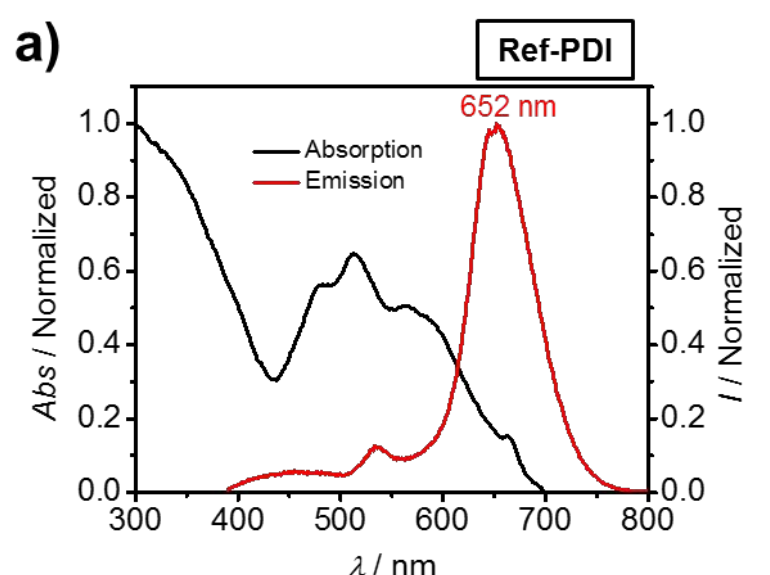

b)
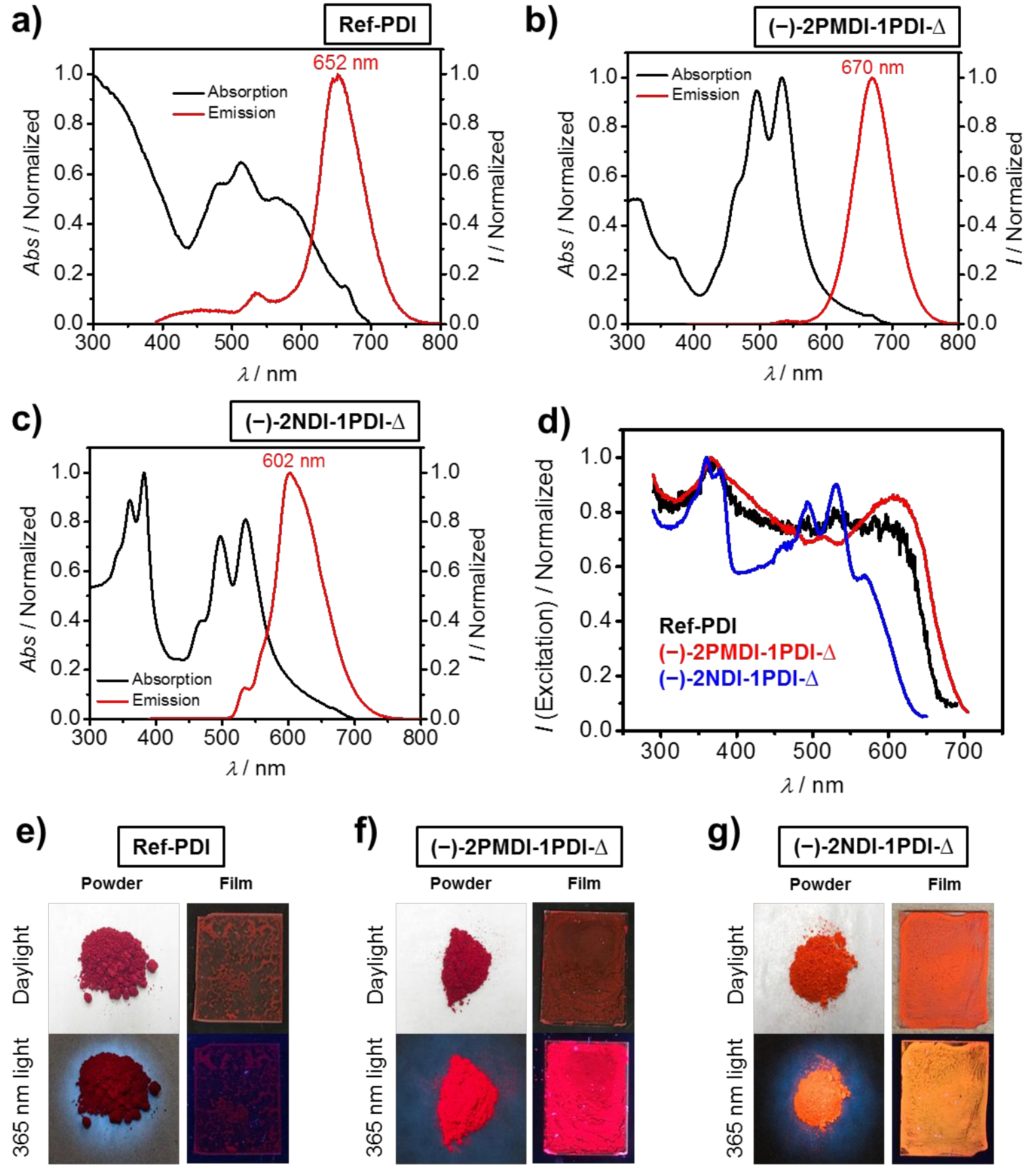

Figure 5 
a)

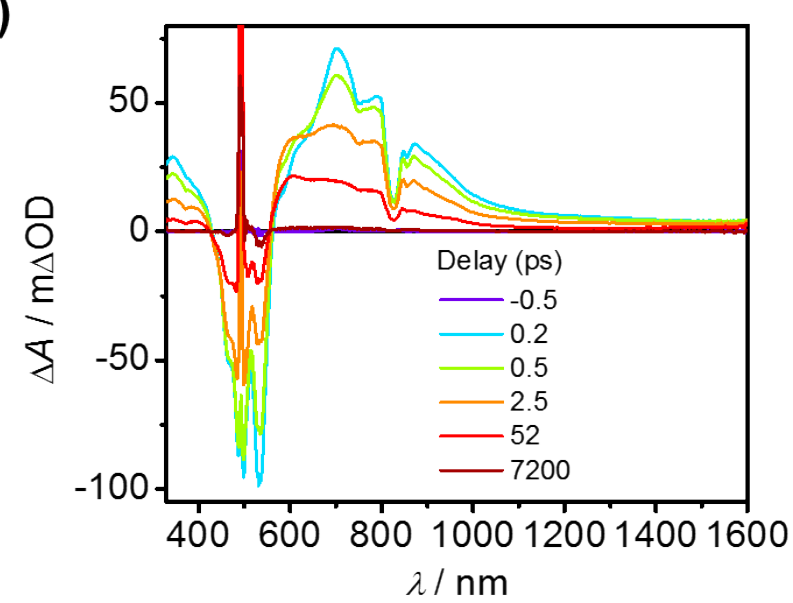

c)

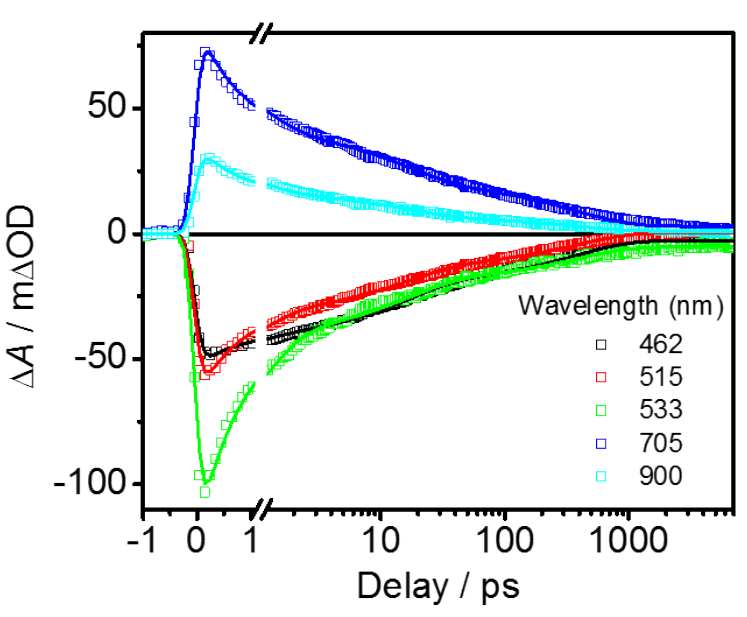

b)

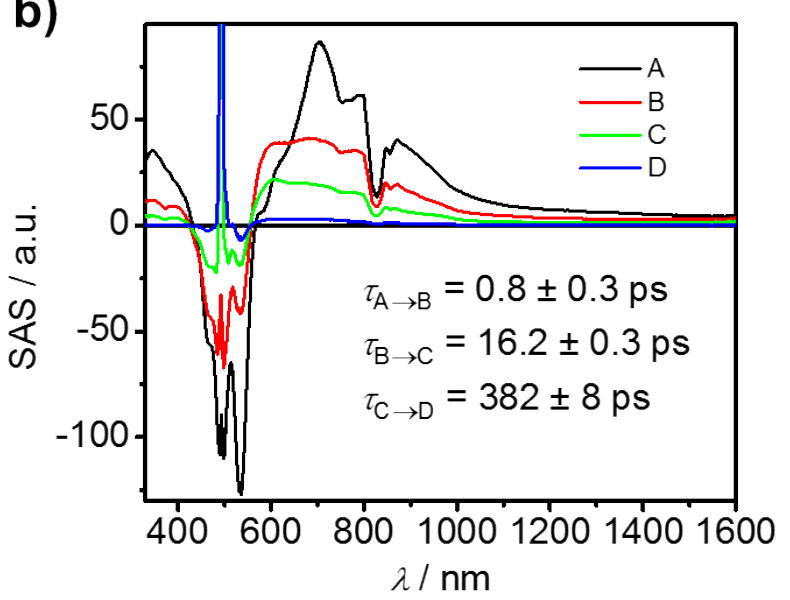

d)

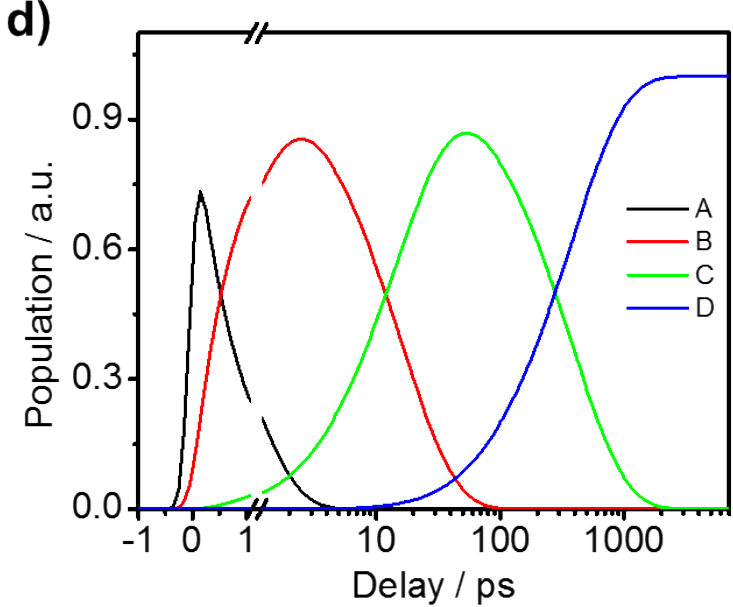

Figure 6 
a)

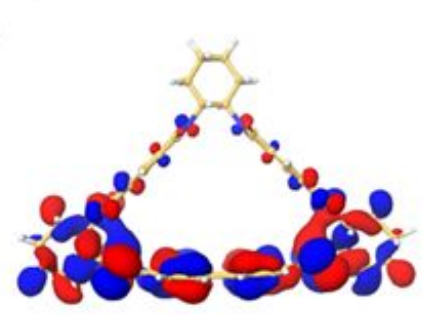

HOMO-2 / $\Delta E=-29.56$

e)

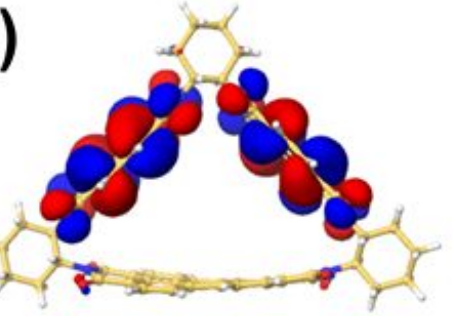

HOMO-2 I $\triangle E=-22.33$

i)

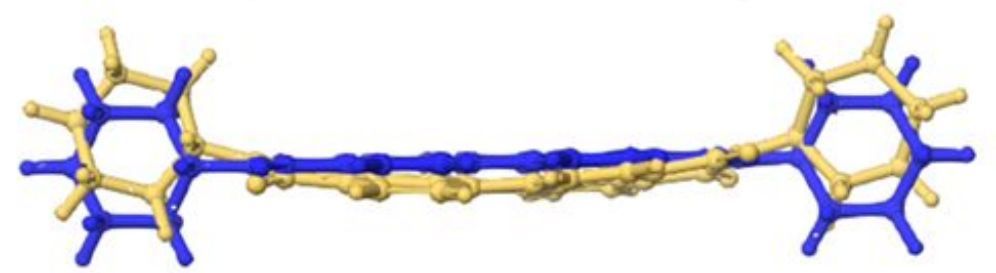

c)

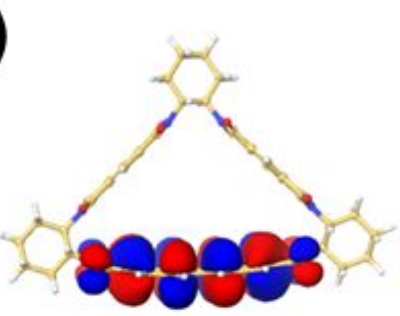

HOMO / $\triangle E=0.0$

f)

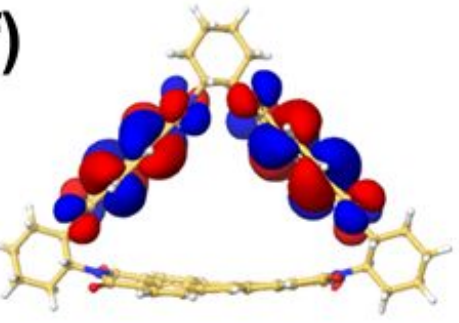

HOMO-1 I $\triangle E=-21.81$ g)

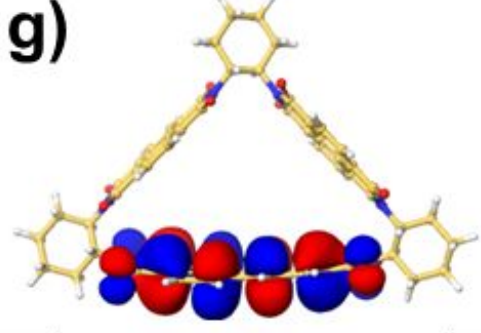

HOMO I $\triangle E=0.0$ d)

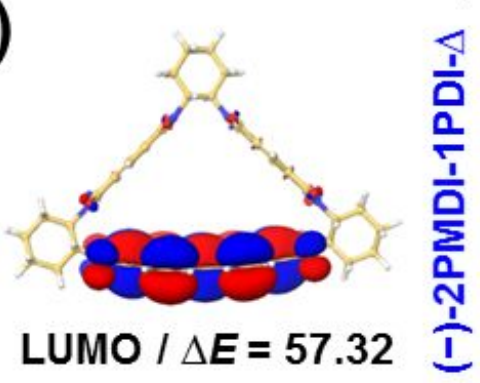

h)

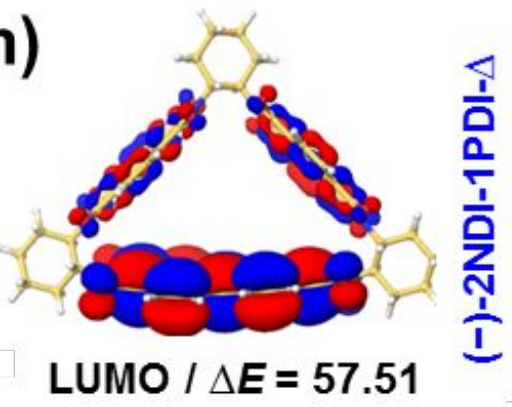

Figure 7 


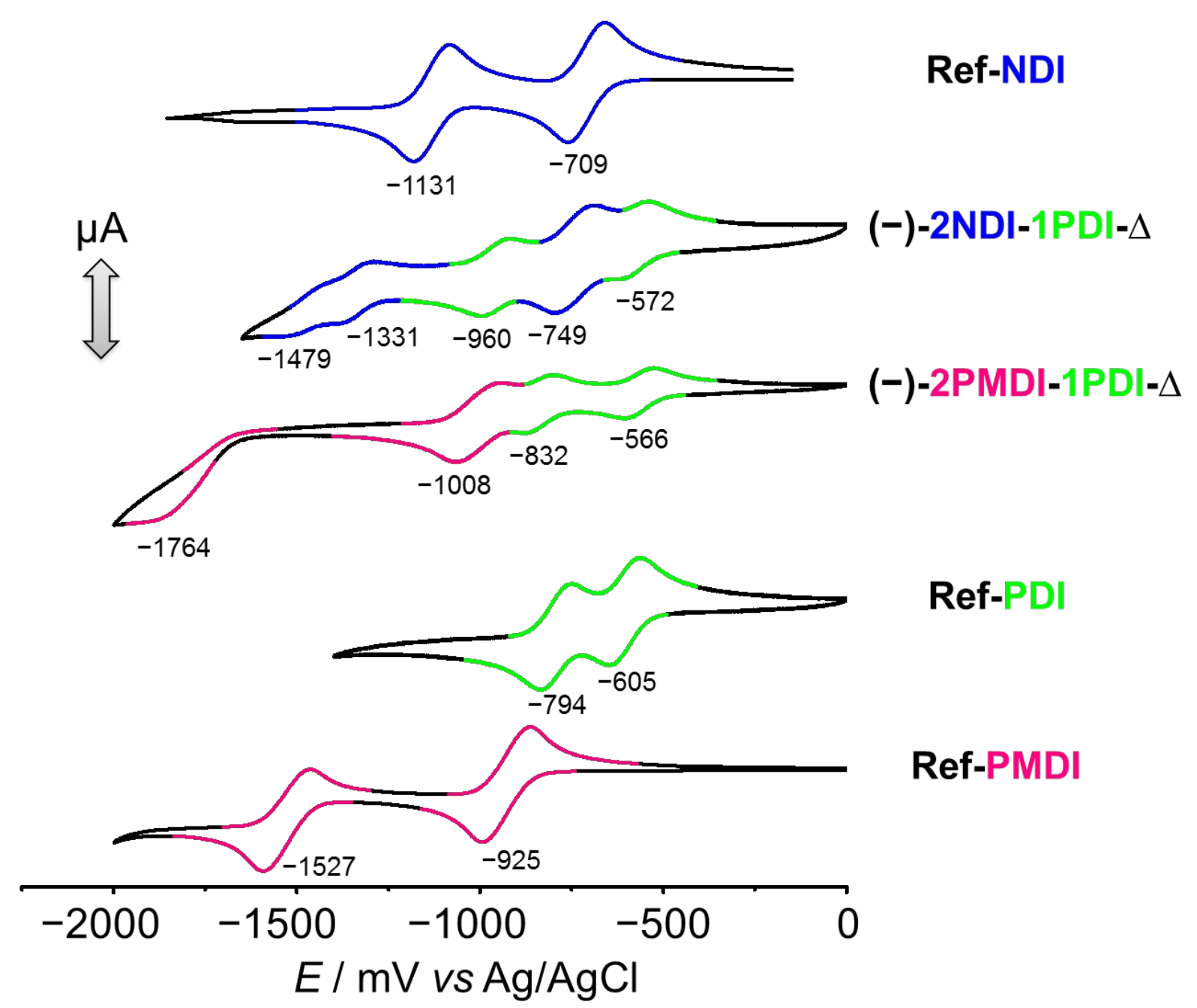

Figure 8 


\section{Table 1. Photophysical properties of reference compound}

\section{and isosceles triangles in $\mathrm{CH}_{2} \mathrm{Cl}_{2}$ at room temperature.}

\begin{tabular}{|c|c|c|c|c|c|c|c|}
\hline \multirow[t]{2}{*}{ Compound } & \multirow{2}{*}{$\begin{array}{c}\text { Absorption } \\
\lambda_{\text {abs }}(\mathbf{n m})\end{array}$} & \multirow[t]{2}{*}{$\varepsilon / \mathbf{M}^{-1} \mathbf{c m}^{-1}$} & \multirow{2}{*}{$\begin{array}{c}\text { Fluorescence } \\
\lambda_{\mathrm{em}}(\mathbf{n m})\end{array}$} & \multicolumn{3}{|c|}{$\begin{array}{c}\text { Quantum yield } \\
\Phi_{\mathrm{f}}(\%)^{\mathrm{a}} \\
\end{array}$} & \multirow{2}{*}{$\begin{array}{c}\text { Lifetime } \\
<\tau_{\text {em }}>\text { (ns) }\end{array}$} \\
\hline & & & & $\mathrm{CH}_{2} \mathrm{Cl}_{2}{ }^{\mathrm{a}}$ & $\mathbf{M e C N}^{b}$ & PhMe $^{\mathrm{b}}$ & \\
\hline Ref-PDI & 524 & 78370 & 530 & 100 & 89 & 94 & 4.0 \\
\hline (-)-2PMDI-1PDI- $\Delta$ & 527 & 61490 & 534 & 100 & 96 & 100 & 4.5 \\
\hline (-)-2NDI-1PDI- $\Delta$ & 527 & 63250 & 534 & 88 & 100 & $63^{c}$ & 5.0 \\
\hline
\end{tabular}

a Relative fluorescence quantum yields in $\mathrm{CH}_{2} \mathrm{Cl}_{2}$ were determined with $N, N$ '-dicyclohexylperylene3,4:9,10-tetracarboxylic acid diimide as a reference under high dilution conditions (within $\pm 3 \%$ error).

${ }^{\mathrm{b}}$ Absolute fluorescence quantum yields were measured in $\mathrm{MeCN}$ and $\mathrm{PhMe}$ using an integrating sphere under high dilution conditions (within $\pm 3 \%$ error).

${ }^{\mathrm{c}}$ Reduced $\Phi_{\mathrm{f}}$ value of (-)-2NDI-1PDI- $\Delta$ in PhMe can be attributed to aggregation-caused quenching.

\section{Table 2. Photophysical properties of reference compound}

\section{and isosceles triangles in the solid state at room}

\section{temperature.}

\begin{tabular}{|c|c|c|c|c|}
\hline \multirow{2}{*}{ Compound } & \multirow{2}{*}{$\begin{array}{c}\text { Photoluminescence } \\
\lambda_{\mathrm{em}}(\mathbf{n m})\end{array}$} & \multicolumn{2}{|c|}{ Quantum yield (\%) $^{\mathbf{a}}$} & \multirow{2}{*}{$\begin{array}{c}\text { Lifetime } \\
<\tau_{\mathrm{em}}>(\mathbf{n s})\end{array}$} \\
\cline { 2 - 4 } & 652 & Powder $\left(\Phi_{\text {powder }}\right)$ & Film $\left(\Phi_{\text {film }}\right)$ & \\
\hline Ref-PDI & 670 & 0.1 & 0.2 & 1.5 \\
\hline (-)-2PMDI-1PDI- $\Delta$ & 602 & 3 & 2 & 13.4 \\
\hline (-)-2NDI-1PDI- $\Delta$ & 4 & 2 & 3.0 \\
\hline
\end{tabular}

a Absolute photoluminescence quantum yields in the solid state (powder form and film state) were determined with an integrating sphere (within $\pm 5 \%$ error). 


\section{Table of Contents}

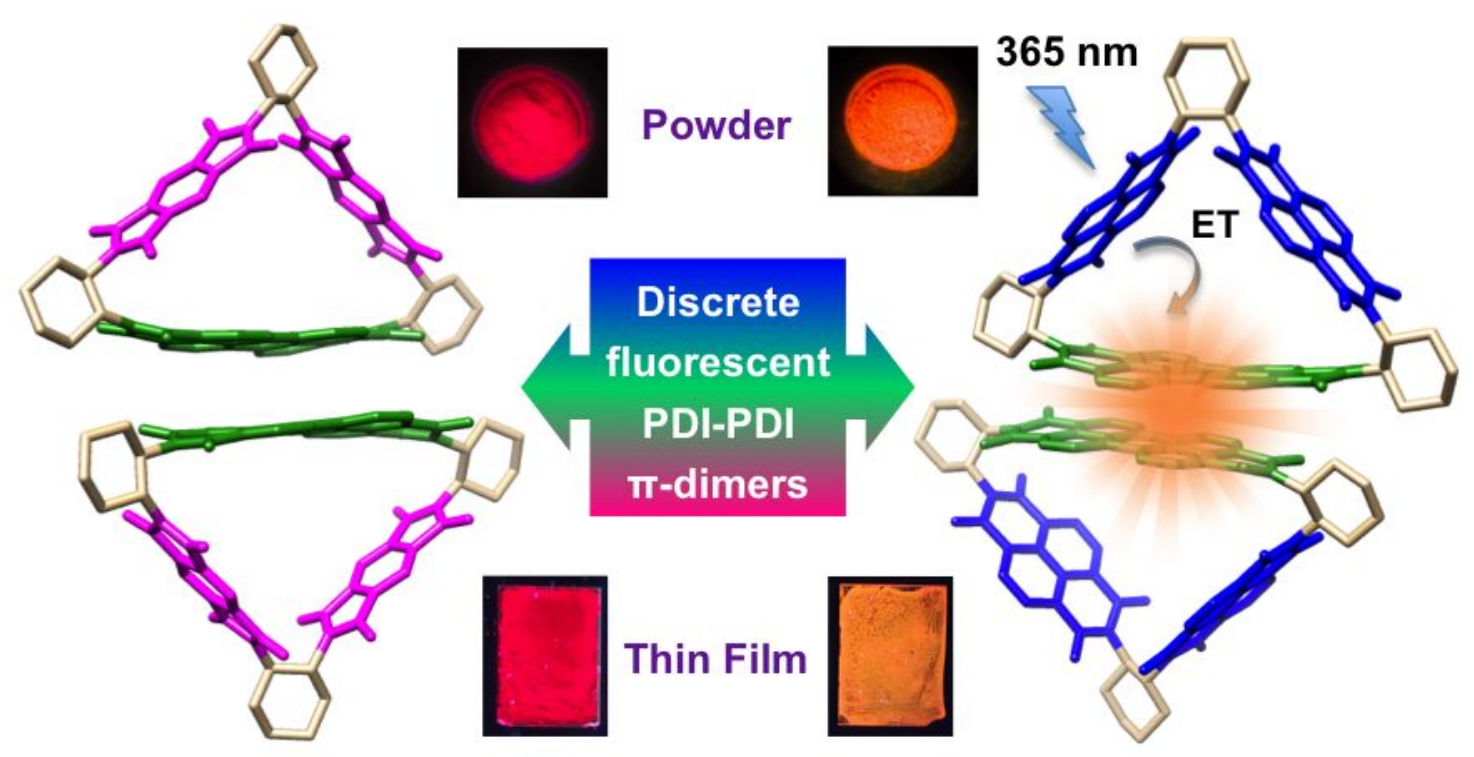

